Full length article

\title{
Combinatory approach for developing silk fibroin scaffolds for cartilage regeneration
}

\author{
Viviana P. Ribeiro ${ }^{\mathrm{a}, \mathrm{b}, *}$, Alain da Silva Morais ${ }^{\mathrm{a}, \mathrm{b}}$, F. Raquel Maia ${ }^{\mathrm{a}, \mathrm{b}}$, Raphael F. Canadas ${ }^{\mathrm{a}, \mathrm{b}}$, João B. Costa ${ }^{\mathrm{a}, \mathrm{b}}$, \\ Ana L. Oliveira ${ }^{\mathrm{c}}$, Joaquim M. Oliveira ${ }^{\mathrm{a}, \mathrm{b}, \mathrm{d}}$, Rui L. Reis ${ }^{\mathrm{a}, \mathrm{b}, \mathrm{d}}$ \\ a 3B's Research Group - Biomaterials, Biodegradables and Biomimetics, University of Minho, Headquarters of the European Institute of Excellence on Tissue Engineering \\ and Regenerative Medicine, AvePark - Parque de Ciência e Tecnologia, Zona Industrial da Gandra, 4805-017 Barco, Guimarães, Portugal \\ ${ }^{\mathrm{b}}$ ICVS/3B's - PT Government Associated Laboratory, Braga/Guimarães, Portugal \\ ${ }^{\mathrm{C}} \mathrm{CBQF}$ - Centro de Biotecnologia e Química Fina, Laboratório Associado, Escola Superior de Biotecnologia, Universidade Católica Portuguesa, Rua Arquiteto Lobão Vital, \\ 4202-401 Porto, Portugal \\ ${ }^{\mathrm{d}}$ The Discoveries Centre for Regenerative and Precision Medicine, Headquarters at University of Minho, Avepark, 4805-017 Barco, Guimarães, Portugal
}

\section{A R T I C L E I N F O}

Article history:

Received 18 December 2017

Received in revised form 13 March 2018

Accepted 28 March 2018

Available online $\mathrm{xxxx}$

\section{Keywords:}

Silk fibroin

Horseradish peroxidase-mediated

crosslinking

Salt-leaching

Freeze-drying

Human adipose-derived stem cells

Articular cartilage

\begin{abstract}
A B S T R A C T
Several processing technologies and engineering strategies have been combined to create scaffolds with superior performance for efficient tissue regeneration. Cartilage tissue is a good example of that, presenting limited self-healing capacity together with a high elasticity and load-bearing properties. In this work, novel porous silk fibroin (SF) scaffolds derived from horseradish peroxidase (HRP)-mediated crosslinking of highly concentrated aqueous SF solution (16 wt\%) in combination with salt-leaching and freeze-drying methodologies were developed for articular cartilage tissue engineering (TE) applications. The HRP-crosslinked SF scaffolds presented high porosity $(89.3 \pm 0.6 \%)$, wide pore distribution and high interconnectivity ( $95.9 \pm 0.8 \%$ ). Moreover, a large swelling capacity and favorable degradation rate were observed up to 30 days, maintaining the porous-like structure and $\beta$-sheet conformational integrity obtained with salt-leaching and freeze-drying processing. The in vitro studies supported human adipose-derived stem cells (hASCs) adhesion, proliferation, and high glycosaminoglycans (GAGs) synthesis under chondrogenic culture conditions. Furthermore, the chondrogenic differentiation of hASCs was assessed by the expression of chondrogenic-related markers (collagen type II, Sox-9 and Aggrecan) and deposition of cartilage-specific extracellular matrix for up to 28 days. The cartilage engineered constructs also presented structural integrity as their mechanical properties were improved after chondrogenic culturing. Subcutaneous implantation of the scaffolds in CD-1 mice demonstrated no necrosis or calcification, and deeply tissue ingrowth. Collectively, the structural properties and biological performance of these porous HRP-crosslinked SF scaffolds make them promising candidates for cartilage regeneration.
\end{abstract}

\section{Statement of Significance}

In cartilage tissue engineering (TE), several processing technologies have been combined to create scaffolds for efficient tissue repair. In our study, we propose novel silk fibroin (SF) scaffolds derived from enzymatically crosslinked SF hydrogels processed by salt-leaching and freeze-drying technologies, for articular cartilage applications. Though these scaffolds, we were able to combine the elastic properties of hydrogel-based systems, with the stability, resilience and controlled porosity of scaffolds processed via salt-leaching and freeze-drying technologies. SF protein has been extensively explored for TE applications, as a result of its mechanical strength, elasticity, biocompatibility, and biodegradability. Thus, the structural, mechanical and biological performance of the proposed scaffolds potentiates their use as three-dimensional matrices for cartilage regeneration.

(c) 2018 Acta Materialia Inc. Published by Elsevier Ltd. All rights reserved.

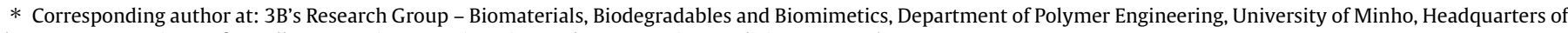
the European Institute of Excellence on Tissue Engineering and Regenerative Medicine, Portugal.

E-mail addresses: viviana.ribeiro@dep.uminho.pt (V.P. Ribeiro), alain.morais@dep.uminho.pt (A. da Silva Morais), raquel.maia@dep.uminho.pt (F.R. Maia), raphael.

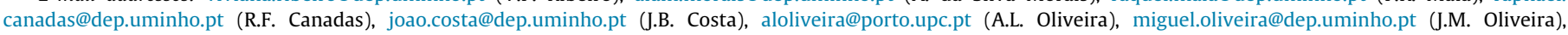
rgreis@dep.uminho.pt (R.L. Reis).
} 


\section{Introduction}

Articular cartilage is a highly-organized tissue with avascular properties and limited intrinsic healing capacity [1]. Injuries and degenerative conditions may compromise cartilage functions and cause pain and disability to the patients. A study performed by Widuchowski et al. [2] involving 25,124 knee arthroscopies showed chondral lesions in $60 \%$ of the cases, classified as focal chondral lesions (67\%), osteoarthritis (29\%), osteochondritis dissecans (2\%) and other types of lesions (1\%). Surgical procedures remain the standard treatment for injured and degenerative joint pathologies, which may include early surgical interventions, such us osteotomy, autologous osteochondral graft transplantation and arthroscopic microfracture chondroplasty, or the total tissue replacement applied in cases of end-stage degenerative pathologies [3]. Thus, the real challenge for orthopaedic surgeons is still the management of articular cartilage defects, whose treatment options are in most cases only for symptom relief and do not ensure the long-term healing of tissue.

Bioactive substitutes emerged over the past years as possible solutions for the treatment and regeneration of articular cartilage tissue [1]. For example, a clinical success has been obtained since 1994 through the use of autologous chondrocytes implantation (ACI) techniques directly at the cartilage defects [4]. Since then, cartilage tissue engineering (TE) strategies have been proposed as cell-free or cell-based approaches, combined with biomimetic materials as supporting matrices [5]. The clinical use of scaffolds in cartilage regeneration is represented only by a few examples of commercially available products, including the Chondro-Gide ${ }^{\circledR}$ (Geistlich Pharma AG, Wolhusen, Switzerland) collagen scaffold that presented promising results for mid-long-term cartilage treatment [6]. The inclusion of interconnected porous-like structures showed to benefit cell adhesion and guided a rapid extracellular matrix (ECM) formation and infiltration within the scaffolds, which represents great advancements on cartilage tissue regeneration, especially when envisioning the use of cell-free strategies $[7,8]$. On the other hand, different polymeric-based hydrogels have been investigated as injectable systems for arthroscopic insertion approaches [9]. The high-water-content that mimics the native cartilage tissue, or the ability to encapsulate cells and diffuse growth factors may benefit this technology [10]. Gelrin $C^{\circledR}$ (Regentis, Haifa, Isreal) and ChonDux ${ }^{\mathrm{TM}}$ (Cartilix, CA, USA) hydrogels composed of polyethylene glycol (PEG) covalently bonded to fibrinogen chains and to hyaluronic acid, respectively, are two good examples of biocompatible and bioabsorbable injectable system that have been successfully used in clinic to fill and reinforce microfractured cartilage defects [11].

Silk fibroin (SF) solutions have been used to prepare hydrogels as injectable or non-injectable systems, with suitable mechanical properties for load bearing of cartilage tissue [12-14]. Most of these hydrogels are formed in a sol-gel transition, together with a structure conformation transition from amorphous to $\beta$-sheet, which can happen by means of different physical and chemical treatments [15]. Different authors have shown that the addition of organic solvents (methanol) [16], decreasing the $\mathrm{pH}$ value [17] or increasing the temperature/ionic species $\left(\mathrm{Ca}^{2+}\right)$ concentration [18] in the aqueous SF solution, can induce SF hydrogels formation with gelation times that can range from a few hours to days, depending on the applied method. Moreover, in cases where cell encapsulation is desired, some of these methods may be compromised. For example, the use of methanol as crosslinking agent imply the immersion of SF solution into the solvent until the reaction is complete and the hydrogel formed. This way, the cell encapsulation process would be compromised, since cells would not survive in the presence of methanol solution. Nevertheless, this process does not invalidate the methanol-induced SF hydrogels for cell-seeding approaches [19]. The use of external stimuli-like ultrasonication and mechanical agitation, have also been explored to produce fast-formed SF hydrogels, compatible for cell encapsulation [20,21]. Also, several enzymatically mediated crosslinking methods have been used to prepare in situ formed hydrogels. Comparing to other systems, enzyme-mediated crosslinking can display several advantages. They occur at physiological conditions and the gelation time can be easily tuned to be fast, favouring cell encapsulation and the delivery of bioactive molecules [22]. Recently, the horseradish peroxidase (HRP) mediated crosslinking has received an increasing interest, since the phenol groups in tyrosine, tyramine or aminophenol, can be conjugated when catalyzed by peroxidase and hydrogen peroxide $\left(\mathrm{H}_{2} \mathrm{O}_{2}\right)[23,24]$. Considering that SF protein contains some reactive amino acid residues of tyrosine $(\sim 5 \%)$, this was seen as a great advantage to create naturally induced SF hydrogels [16,25]. These HRP-mediated crosslinked SF hydrogels were previously produced in a main amorphous conformation and at physiological conditions, showing promising biological results and tunable mechanical properties according to the protein/crosslinker concentration. Simultaneously, it has been recognized that the anti-parallel $\beta$-sheets formation in SF hydrogels or scaffolds can affect their stiffness, degradability and biological behavior in response to changes in surface roughness [26]. For example, Yan et al. [27] combined salt-leaching and freeze-drying methodologies to produce macro/microporous SF scaffolds, showing that the anti-parallel $\beta$-sheet conformation was present in the scaffolds and improved their mechanical properties for cartilage TE scaffolding strategies. Moreover, high and controlled porosity induced by the saltleaching technique have also shown to improve cell adhesion, proliferation and chondrogenic differentiation within SF constructs [14].

On the side of the cells, the combination of three-dimensional (3D) networks with autologous chondrocytes previous implantation, represented significant clinical improvements for cartilage defects treatment (see NeoCart ${ }^{\circledR}$, Histogenics, Waltham, MA and Hyalograft $^{\circledR}$ C, Anika Therapeutics, Bedford, MA) [28,29]. Nevertheless, great attention has been directed for cell sources able to differentiate into the chondrogenic lineage, and secret growth factors responsible for creating an ECM that can fill the chondral lesion [30]. The application of human mesenchymal stem cells (MSCs) covered a noticeable interest having shown to possess multilineage differentiation potential including for chondrogenesis induction [31]. On the other hand, human adipose-derived stem cells (hASCs) appear to present the same multilineage differentiation potential as MSCs, but also demonstrated special features regarding cell surface markers and abundance in the body. Moreover, they can be easily harvested from the adipose tissue with less pain for the donor [32]. Previous studies, were able to explore the chondrogenic differentiation potential of hASCs when cultured in SF scaffold materials, showing that the 3D porous matrices successfully induced cell-cell interactions and ECM matrix formation in a similar fashion to the forming cartilage tissue [33,34].

In the present work, we aimed to make use of an enzymaticmediated approach to crosslink SF combined with salt-leaching and freeze-drying technologies, towards producing robust and interconnected porous SF scaffolds for cartilage TE applications. With this strategy, it is expected to tune a large number of properties in a single HRP-crosslinked SF scaffold, while avoiding the use of harsh or toxic crosslinking agents during processing. These work reports on the effectiveness of the proposed scaffolds over in vitro chondrogenic differentiation and in vivo compatibility, being the first study to propose such combinatory approaches for the development of a more complete SF scaffold able to better recreate the requirements for the treatment of cartilage defects. 


\section{Materials and methods}

\subsection{Materials and reagents}

Silk derived from the silkworm Bombyx mori in the form of cocoons was provided by the Portuguese Association of Parents and Friends of Mentally Disabled Citizens (APPACDM, Castelo Branco, Portugal). Silicone tubing was purchased from Deltalab (Barcelona, Spain). Granular sodium chloride (Commercial grade, Portugal) was prepared in the range of $500-1000 \mu \mathrm{m}$ in an analytic sieve shaker (AS 200 Digit, Retsch, Germany). All reagents were purchased from Sigma-Aldrich (St. Louis, MO, USA) unless otherwise stated.

\subsection{Preparation of aqueous silk fibroin solution}

Purified silk fibroin (SF) was prepared by removing the glue-like protein sericine from the cocoons in a boiling sodium carbonate solution $(0.02 \mathrm{M})$ for $1 \mathrm{~h}$ and then rinsed with distilled water in order to fully remove the degumming solution. A $9.3 \mathrm{M}$ lithium bromide solution was used to dissolve the purified SF for $1 \mathrm{~h}$ at $70{ }^{\circ} \mathrm{C}$ and dialyzed in distilled water for $48 \mathrm{~h}$ using a benzoylated dialysis tubing (MWCO: $2 \mathrm{kDa}$ ). Next, the aqueous SF solution was concentrated using a $20 \mathrm{wt} \%$ poly(ethylene glycol) solution for at least $6 \mathrm{~h}$. The final concentration of SF (18-20 wt\%) was determined by measuring the dry weight of the SF solution placed in the oven at $70{ }^{\circ} \mathrm{C}$ overnight. Meanwhile, the prepared SF solution was stored at $4{ }^{\circ} \mathrm{C}$ until further use (Fig. 1a).

\subsection{Preparation of salt-leached enzymatically crosslinked silk fibroin scaffolds}

Enzymatically crosslinked SF scaffolds were prepared by the initial mixture of SF solution, diluted to $16 \mathrm{wt} \%$, with horseradish peroxidase solution (HRP type VI, $0.84 \mathrm{mg} / \mathrm{mL}$ ) and hydrogen peroxide solution $\left(\mathrm{H}_{2} \mathrm{O}_{2}, 0.36 \mathrm{wt} \%\right.$; Panreac, Barcelona, Spain), as reported previously [16]. Briefly, $1 \mathrm{~mL}$ SF solution, $50 \mu \mathrm{L}$ HRP solution and $65 \mu \mathrm{L} \mathrm{H}_{2} \mathrm{O}_{2}$ solution $(1 / 0.26 \% / 1.45 \%)$ were mixed in a $1.5 \mathrm{~mL}$ centrifuge tube (Eppendorf, Hamburg, Germany) and before gelling, $1 \mathrm{~mL}$ of the $\mathrm{SF} / \mathrm{HRP} / \mathrm{H}_{2} \mathrm{O}_{2}$ solution was transferred into a cylindrical shaped silicone mold ( $9 \mathrm{~mm}$ inner diameter). $\beta$-sheet induced SF structures were prepared by the slow addition of $2 \mathrm{~g}$ of granular sodium chloride (particle size $500-1000 \mu \mathrm{m}$ ) to the solution. The sodium chloride particles were gently added to the silicon molds to facilitate the precipitation of the salt [27]. The molds were placed in the oven at $37^{\circ} \mathrm{C}$ to complete the enzymatic reaction at the $\mathrm{SF} / \mathrm{HRP} / \mathrm{H}_{2} \mathrm{O}_{2}$ complex. HRP-crosslinked SF scaffolds were formed when the undissolved sodium chloride particles was leached out (porogen) in distilled water for $72 \mathrm{~h}$. Finally, the scaffolds were removed from the molds by using a biopsy punch $(8 \mathrm{~mm}$ inner diameter; Smith \& Nephew, Portugal), followed by freezing

a

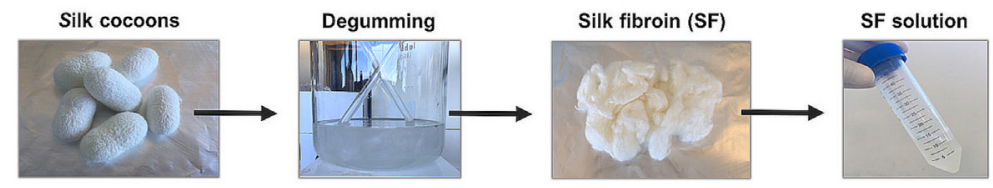

b

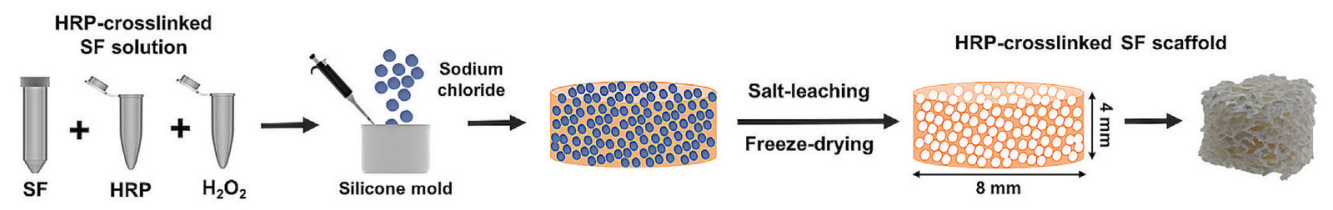

C

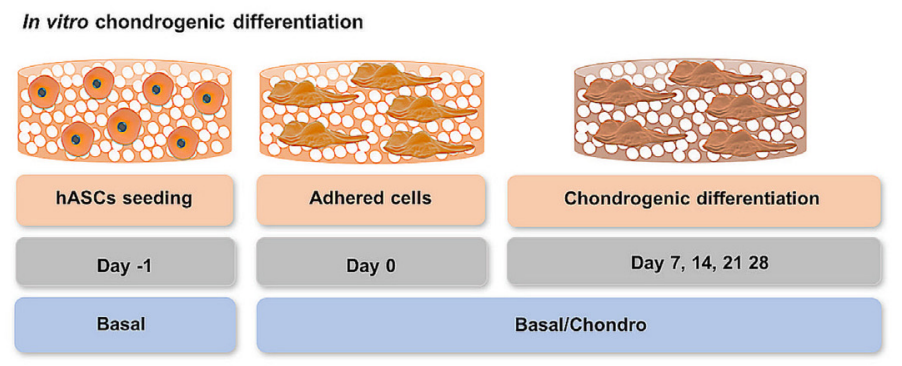

d

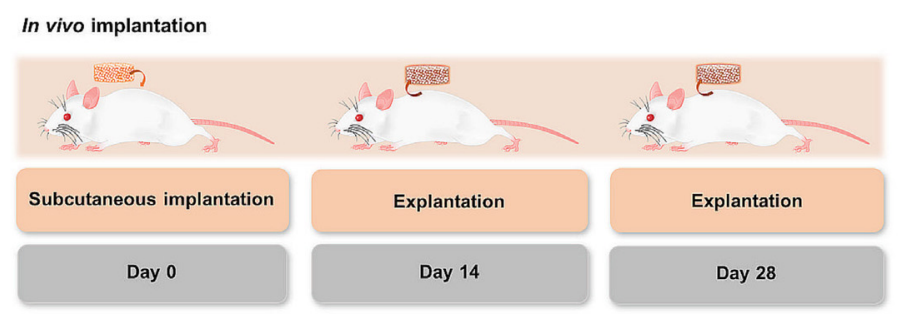

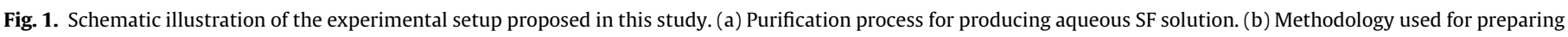

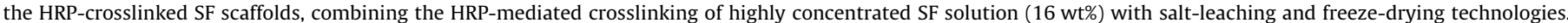

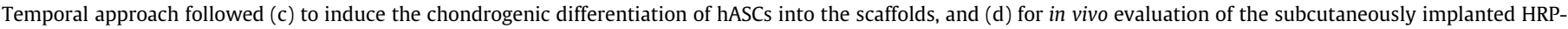
crosslinked SF scaffolds. The diameter and height of scaffolds were $8 \mathrm{~mm}$ and $4 \mathrm{~mm}$, respectively. 
at $-80^{\circ} \mathrm{C}$ overnight and freeze-drying (Telstar Cryodos-80, Barcelona, Spain) for 7 days (Fig. 1b).

\subsection{Physicochemical characterization}

\subsubsection{Scanning electron microscopy}

The surface and cross-sectional morphology of the HRPcrosslinked SF scaffolds was analyzed by analytic scanning electron microscopy (SEM) using a JEOL JSM-6010LV (Tokyo, Japan). Two samples were used for structure qualitative evaluation. Prior analysis all samples were sputter-coated with gold using a Leica EM ACE600 coater (Leica Microsystems, Wien, Austria).

\subsubsection{Micro-computed tomography}

The microstructure of the prepared HRP-crosslinked SF scaffolds was evaluated using a high-resolution X-ray microtomography system Skyscan 1072 scanner (Skyscan, Kontich, Belgium). Samples were scanned using a pixel size of $8.79 \mu \mathrm{m} \mathrm{x} / \mathrm{y} / \mathrm{z}$ and an $\mathrm{X}$-ray source fixed at $40 \mathrm{keV}$ of energy and $248 \mu \mathrm{A}$ of current. After acquisition, reconstructed grey-scale images were converted into binary images by using a dynamic threshold of $40-255$. Then, the binary images were used for morphometric analysis (CT Analyzer v1.12.0.0, SkyScan, Kontich, Belgium) by quantification of porosity, mean pore size, mean wall thickness and interconnectivity. The scaffolds cross-sectional images and 3D virtual models were also created, visualized and registered using the image processing and reconstruction software's Data Viewer (v1.6.6.0) and CT-Vox (v2.0.0) (SkyScan, Kontich, Belgium), respectively. Three samples were used for the qualitative and quantitative microstructure evaluation.

\subsubsection{Fourier transform infrared spectroscopy}

The infrared spectra of the HRP-crosslinked SF scaffolds were recorded by Fourier transform infrared (FTIR) spectroscopy (Perkin-Elmer 1600 series equipment, CA, USA) under an attenuated total reflectance (ATR) model (IRPrestige-21, Shimadzu, Kyoto, Japan), equipped with a germanium crystal. Prior analysis, the samples were immersed in phosphate-buffered saline (PBS, $\mathrm{pH}$ 7.4) solution until equilibrium was reached (overnight). All spectra were obtained between 4500 and $800 \mathrm{~cm}^{-1}$ at a $4 \mathrm{~cm}^{-1}$ resolution with 32 scans. Three samples were used for analysis and PBS was used as background.

\subsubsection{Compressive tests}

The mechanical behavior of the HRP-crosslinked SF scaffolds was tested under quasi-static compression in dry and hydrated state using a Universal Testing Machine (Instron 4505, Norwood, MA, USA). The diameter and height of the scaffolds were 8 and $4 \mathrm{~mm}$, respectively. Prior analysis, the samples tested in hydrated state were immersed in PBS solution ( $\mathrm{pH}$ 7.4) until equilibrium was reached (overnight). The cross-head speed was fixed at $2 \mathrm{~mm} / \mathrm{min}$ and tests were run until a $60 \%$ reduction in sample height had been achieved. The compressive stress and strain were graphed and the average compressive modulus and compressive strength were determined based on five samples measured for each group. The elastic modulus (E) or Young's modulus for compression was defined by the slope of the initial linear region of the stress-strain curve, described as elastic deformation region. A plastic deformation state was reached in the following linear region, above the yield point, until the densification and complete collapse of material at the final region of the stress-strain curve, in which the stress rises substantially. The compressive strength was determined by the maximum compressive load $(\mathrm{N})$ applied in the test divided by the original area of the specimen $\left(\mathrm{mm}^{2}\right)$.

\subsection{Swelling ratio and degradation profile}

The swelling ratio of the HRP-crosslinked SF scaffolds was tested in PBS solution. The initial dry weight of each sample $(n=6)$ was measured, and then the scaffolds were immersed in $5 \mathrm{~mL}$ of PBS solution. The study was conducted at $37^{\circ} \mathrm{C}$ for a time period ranging from $1 \mathrm{~h}$ to 30 days. The solutions were changed weekly. At the end of each time point, samples were gently blotted with filter paper to remove excess liquid and the wet weight measured. The swelling ratio was determined as:

Water uptake $(\%)=\frac{m w-m i}{m i} \times 100$

where $m_{w}$ is the weight of the sample after removal from the solution and $m_{i}$ is the initial weight of the sample.

The stability of the HRP-crosslinked SF scaffolds was evaluated by enzymatic degradation test. Protease XIV was derived from Streptomyces griseus $(3.5 \mathrm{U} / \mathrm{mg}$ ) and prepared at $3.5 \mathrm{U} / \mathrm{mL}$ and $0.2 \mathrm{U} / \mathrm{mL}$ by dissolving the enzyme in PBS [35]. As control condition, samples were incubated in PBS solution alone. The initial dry weight of each sample $(n=3)$ was measured, and then the scaffolds were immersed in $5 \mathrm{~mL}$ of protease solution or PBS solution. The study was conducted at $37^{\circ} \mathrm{C}$ for a time period ranging from $3 \mathrm{~h}$ to 30 days. The enzyme solutions were changed every $24 \mathrm{~h}$. The dry weight was measured after washing the samples with distilled water and drying at $70^{\circ} \mathrm{C}$ overnight. The weight loss was determined as:

Weight loss $(\%)=\frac{m i-m f}{m i} \times 100$

where $m_{i}$ is the initial weight of the sample and $m_{f}$ is the weight of the sample after drying.

\subsection{In vitro cell studies}

\subsection{1. hASCs isolation and expansion}

Human abdominal subcutaneous adipose tissue samples were obtained from lipoaspiration procedures performed on healthy male and female donors with ages between 18 and 57 years, after informed consent, under established cooperative agreements between Hospital da Senhora da Oliveira (Guimarães, Portugal) and 3B's Research Group. All the samples were processed within $24 \mathrm{~h}$ after the lipoaspiration procedure and human adiposederived stem cells (hASCs) were enzymatically isolated from subcutaneous adipose tissue as previously described [36]. Human ASCs were expanded in basal medium consisting of MEM alpha medium ( $\alpha$-MEM; Gibco ${ }^{\circledR}$, Life Technologies, Carlsbad, CA, USA) supplemented with sodium bicarbonate, $10 \%(\mathrm{v} / \mathrm{v})$ fetal bovine serum (FBS; Life Technologies, Carlsbad, CA, USA) and $1 \%(\mathrm{v} / \mathrm{v})$ antibiotic/antimycotic solution (final concentration of penicillin 100 units $/ \mathrm{mL}$ and streptomycin $100 \mathrm{mg} / \mathrm{mL}$, Life Technologies, Carlsbad, CA, USA). Cells were cultured until confluence at $37^{\circ} \mathrm{C}$, $5 \% \mathrm{CO}_{2}$ incubator, changing the culture medium every $2-3$ days.

\subsubsection{Seeding and chondrogenic differentiation of hASCs on the HRP- crosslinked SF scaffolds}

Human ASCs were characterized by flow cytometry (FACSCalibur flow cytometer, BD Biosciences, San Jose, CA, USA) for MSCs surface markers [37]. The following monoclonal antibodies conjugated to fluorochromes were used: anti-CD105 FITC (BioRad, Hercules, CA, USA), anti-CD73 PE, anti-CD90 APC, anti-CD45 FITC, anti-CD34 PE (BD Biosciences, San Jose, CA, USA). HRPcrosslinked SF scaffolds of $8 \mathrm{~mm}$ in diameter and $4 \mathrm{~mm}$ in height were used for the in vitro studies and sterilized by ethylene oxide (Gas Sterilizer/Aerator, Steri-Vac 5XL, 3 M company, St. Paul, MN, USA). The number of cell-seeded constructs used for the different 
characterization techniques will be described below. Before cell seeding, all scaffolds were hydrated in Dulbecco's modified Eagle's medium-high glucose (DMEM-HG; Gibco ${ }^{\circledR}$, Life Technologies, Carlsbad, CA, USA) supplemented with $1 \%(\mathrm{v} / \mathrm{v})$ antibiotic/antimycotic solution, overnight in the $\mathrm{CO}_{2}$ incubator. In the following day, the hydrated scaffolds were transferred to 48-well suspension cell culture plates (Cellstar, Greiner Bio-One, Kremsmuenster Austria). Confluent hASCs (passage 2) were detached with TrypLE ${ }^{\mathrm{TM}}$ Express (Life Technologies, Carlsbad, CA, USA) and seeded in a $20 \mu \mathrm{L}$ cell suspension onto the surface of the scaffolds, at a density of $3 \times 10^{5}$ cells/scaffold. The constructs were kept in the $\mathrm{CO}_{2}$ incubator for $3 \mathrm{~h}$ and then completed with $1 \mathrm{~mL}$ of DMEM-HG supplemented with $10 \%$ (v/v) FBS (Life Technologies, Carlsbad, CA, USA) and $1 \%(\mathrm{v} / \mathrm{v})$ antibiotic/antimycotic solution. After $24 \mathrm{~h}$, the culture medium was refreshed or replaced by chondrogenic differentiation medium. The chondrogenic medium was based on DMEM-HG supplemented with $50 \mu \mathrm{g} / \mathrm{mL}$ Ascorbic acid, $40 \mu \mathrm{g} / \mathrm{mL}$ L-Prolin, $1 \%$ Insulin-Transferrin-Selenium-G (ITS-G; Life Technologies, Carlsbad, CA, USA), $100 \mathrm{nM}$ Dexamethasone, $10 \mathrm{ng} / \mathrm{mL}$ recombinant human TGF- $\beta 1$ (PeproTech, London, UK) and 1\% (v/v) antibiotic/ antimycotic solution. Samples cultured in chondrogenic and basal culture medium, were harvested after culturing for $1,7,14,21$ and 28 days and the culture medium was changed every 2-3 days (Fig. 1c).

\subsubsection{Live/Dead staining}

Cell viability at the HRP-crosslinked SF constructs was confirmed by performing a calcein-AM and propidium iodide (PI; Life Technologies, Carlsbad, CA, USA) staining. At the end of each time-point, three samples of each group (chondrogenic and basal medium) were incubated in $1 \mu \mathrm{g} / \mathrm{mL}$ calcein-AM and $5 \mu \mathrm{g} / \mathrm{mL}$ PI prepared in DMEM-low glucose supplemented with sodium bicarbonate and $1 \%(\mathrm{v} / \mathrm{v})$ antibiotic/antimycotic solution, for $30 \mathrm{~min}$ in the dark at $37^{\circ} \mathrm{C}$ in the $\mathrm{CO}_{2}$ incubator. After washing in PBS, samples were immediately examined under fluorescence microscopy (Calcein-AM in green: ex/em 495/515 nm; PI in red: ex/em $495 / 635 \mathrm{~nm}$ ) in a transmitted and reflected light microscope (Axio Imager Z1 m; Zeiss, Jena, Germany). Images were acquired using the Zen microscope processing software (Zeiss, Jena, Germany) connected to a digital camera AxioCam MR3 (Zeiss, Jena, Germany). A Z-stack function was used to combine images at different depths into one final image.

\subsubsection{Alamar blue assay}

Alamar blue assay was performed to assess the metabolic activity of cells at the HRP-crosslinked SF constructs, following the manufacturer's instructions. After each culture time, the constructs were transferred to a new 48-well suspension cell culture plate and a solution of $10 \%(\mathrm{v} / \mathrm{v})$ AlamarBlue $^{\circledR}$ (BioRad, Hercules, CA, USA), prepared in chondrogenic or basal culture medium, was transferred to the culture plates in $500 \mu \mathrm{L} /$ scaffold. After $3 \mathrm{~h}$ of reaction with cells at $37^{\circ} \mathrm{C}$ in the $\mathrm{CO}_{2}$ incubator, $100 \mu \mathrm{L}$ of Alamar blue solution were taken from each well and placed in a 96-well white opaque plate (Corning-Costar Corporation, Acton, MA, USA) in triplicate. The fluorescence was measured in a microplate reader (Synergy HT, BioTek, Instruments, USA) at an excitation wavelength of 530/25 nm and at an emission wavelength of 590/35 nm. Three samples of each group (chondrogenic and basal medium) were tested at each time-point, in three independent experiments. Scaffolds without cells were used as control.

\subsubsection{Quantitative DNA and glycosaminoglycans assays}

The amount of double stranded DNA (dsDNA), that is directly proportional to the cell number, was determined using a QuantIT PicoGreen dsDNA quantification kit (Life Technologies, Carlsbad, CA, USA), according to the manufacturer's instructions. After each culture time, samples were washed with PBS solution and frozen at $-80{ }^{\circ} \mathrm{C}$ in $1 \mathrm{~mL}$ of ultrapure water until further analysis. To induce complete membrane lysis, samples were sonicated for $1 \mathrm{~h}$ after thawed at room temperature (RT). Supernatant fluorescence was measured using a microplate reader at an excitation wavelength of $485 / 20 \mathrm{~nm}$ and at an emission wavelength of 528/20 nm. The standard curve for DNA analysis was prepared with concentrations ranging from 0 to $2 \mu \mathrm{g} / \mathrm{mL}$.

A dimethylmethylene blue (DMB) assay was performed for glycosaminoglycans (GAGs) quantification on the same samples used for dsDNA quantification. Samples were digested overnight at 60 ${ }^{\circ} \mathrm{C}$ in $1 \mathrm{~mL}$ papain digestion solution, prepared by adding to each $50 \mathrm{~mL}$ of digestion buffer, $25 \mathrm{mg}$ of papain and $48 \mathrm{mg}$ of n-acetyl cysteine. Digestion buffer was composed of $200 \mathrm{mM}$ of phosphate buffer (sodium phosphate monobasic) containing $1 \mathrm{mM}$ ethylenediaminetetraacetic acid (EDTA) (pH 6.8). Then, samples were centrifuged at $13,000 \mathrm{rpm}$ for $10 \mathrm{~min}$ and the supernatants collected. GAGs content was spectrophotometrically determined with a DMB solution, prepared by dissolving $16 \mathrm{mg}$ DMB powder in 900 $\mathrm{mL}$ distilled water containing $3.04 \mathrm{~g}$ glycine and $2.73 \mathrm{~g}$ of $\mathrm{NaCl}$ (Panreac Química SAU, Barcelona, Spain) ( $\mathrm{pH}$ 3.0). The optical density (OD) was measured in a microplate reader at an absorbance of $530 \mathrm{~nm}$. A chondroitin sulphate stock solution was prepared in distilled water at $50 \mu \mathrm{g} / \mathrm{mL}$ in order to make a standard curve with concentrations ranging from 0 to $35 \mu \mathrm{g} / \mathrm{mL}$. The obtained GAGs concentrations were normalized against the DNA concentrations of the same samples. Three samples of each group (chondrogenic and basal medium) were tested at each time-point, in three independent experiments. Scaffolds without cells were used as control.

\subsubsection{Collagenous proteins quantification}

The presence of collagen within the ECM was determined by the Sirius red/Fast green collagen staining kit (Chondrex, Redmond, WA, USA), where the Sirius red dye specifically binds to collagen and Fast green dye stains the non-collagenous proteins. After each culture time, samples were washed with PBS solution and fixed with $10 \%(\mathrm{v} / \mathrm{v})$ formalin (Thermo Scientific, Waltham, MA, USA) for $20 \mathrm{~min}$ at RT, washed again with PBS solution and stored at 4 ${ }^{\circ} \mathrm{C}$ until further analysis. A mixture of $0.1 \%(\mathrm{v} / \mathrm{v})$ Sirius red and $0.1 \%(\mathrm{v} / \mathrm{v})$ Fast green solution saturated with picric acid was transferred to the culture plates in $500 \mu \mathrm{L} / \mathrm{scaffold}$. After $30 \mathrm{~min}$ of incubation at RT, the dye solution was carefully removed and samples were washed with distilled water. Afterwards, the dyes were extracted from the stained samples by applying $2 \mathrm{~mL}$ of dye extraction buffer $(0.05 \mathrm{M} \mathrm{NaOH}$ solution in methanol). The OD was measured in a microplate reader at an absorbance of $540 \mathrm{~nm}$ and $605 \mathrm{~nm}$. The amount of collagenous proteins was calculated according to the manufacturer's instructions. Three samples of each group (chondrogenic and basal medium) were tested at each time-point, in three independent experiments. Scaffolds without cells were used as control.

\subsubsection{RNA isolation and real-time reverse transcriptase-polymerase chain reaction}

The expression of mRNA for the genes of interest on the HRPcrosslinked SF constructs was measured by real-time PCR analysis. Total RNA was extracted from cells recovered from the 3D cultures using Direct-zol ${ }^{\mathrm{TM}}$ RNA MiniPrep kit (Zymo Research, Irvine, CA, USA), following the manufacturer's instructions. After each culture time, samples were washed with PBS solution, immersed in $700 \mu \mathrm{L}$ TRI Reagent ${ }^{\circledR}$ and stored at $-80^{\circ} \mathrm{C}$ until further use. Samples were thawed at RT and sonicated for $15 \mathrm{~min}$ at $20^{\circ} \mathrm{C}$ to ensure the complete lysing of the cells. RNA quantification and purity were assessed using a NanoDrop ND-1000 spectrophotometer (NanoDrop Technologies, Wilmington, DE, USA). Samples with a 260/280 ratio between 1.6 and 2.0 were used for first-strand 
complementary DNA (cDNA) synthesis according to the protocol from the qScript ${ }^{\mathrm{TM}}$ CDNA synthesis Kit (Quanta Biosciences, Gaithersberg, MD, USA) in a MiniOpticon real-time PCR detection system (BioRad, Hercules, CA, USA). Briefly, $100 \mathrm{ng}$ of the total RNA were used to generate single-stranded cDNA by random priming with qScript Reverse Transcriptase (RT). Afterwards, the obtained cDNA was used as template for the amplification of the target genes using the PerfeCta SYBR Green FastMix kit (Quanta Biosciences, Gaithersberg, MD, USA) according to the manufacturer's instructions. The primers sequence, specific for each gene, namely glyceraldehyde-3-phosphate dehydrogenase (GAPDH), collagen type II (Col II), Sox-9 and Aggrecan (ACAN) are described in Table 1. Each real-time PCR run was carried out with an initial incubation at $95^{\circ} \mathrm{C}$ for $2 \mathrm{~min}$, followed by 45 cycles of denaturation $\left(95^{\circ} \mathrm{C}, 10 \mathrm{~s}\right.$ ), annealing (specific for each gene, $25 \mathrm{~s}$ ) and extension $\left(72{ }^{\circ} \mathrm{C}, 30 \mathrm{~s}\right)$ in a Mastercycler ${ }^{\circledR}$ ep realplex real-time PCR system (Eppendorf, Hamburg, Germany). The transcripts expression data were normalized to the endogenous housekeeping gene GAPDH and the relative quantification was calculated using as calibrators the results obtained for each target gene at day 1 in basal culture conditions, according to the Livak $\left(2^{-\Delta \Delta \mathrm{CT}}\right)$ method [38]. Three samples of each group (chondrogenic and basal medium) were tested at each time-point, in three independent experiments.

\subsubsection{Histology and immunofluorescence staining}

At the end of each time-point, three HRP-crosslinked SF constructs from each group (chondrogenic and basal medium) were fixed with $10 \%(\mathrm{v} / \mathrm{v})$ formalin for $20 \mathrm{~min}$ at RT and transferred to histological cassettes for paraffin-embedding. Samples were then serially sectioned with $20 \mu \mathrm{m}$ thick using a microtome (Spencer 820, American Optical Company, NY, USA). Representative sections of the constructs were stained with standard Masson's trichome, using a Bio-Optica staining kit (Milan, Italy) to detect the fibrous collagen content. Sirius red/Fast green collagen kit was also applied as described in 2.6.6, for staining the collagen content with Sirius red dye and counterstaining the non-collagenous proteins with Fast green. Sections were also stained with Alcian blue 8GX (1\% $\mathrm{v} / \mathrm{v}$ in $0.5 \mathrm{M}$ acetic acid glacial; VWR BDH Prolabo, Briare, France) and Safranin-O $(0.1 \% \mathrm{v} / \mathrm{v}$; Honeywell Fluka, Morris Plains, NJ, USA) to detect the distribution and content of sulfated GAGs. For Safranin-O staining, sections were counterstained with Gill-2 hematoxylin (Thermo Scientific, Waltham, MA, USA) and Fast green $(0.02 \%$ v/v; Honeywell Fluka, Morris Plains, NJ, USA).

To detect protein expression, antigen retrieval was performed on the sections by incubating in $0.5 \%$ pepsin in $5 \mathrm{mM} \mathrm{HCl}$ (Fisher Scientific, Waltham, MA, USA) for $30 \mathrm{~min}$ at $37^{\circ} \mathrm{C}$. The sections were permeabilized with $1 \%(\mathrm{v} / \mathrm{v})$ Triton X-100/PBS for $10 \mathrm{~min}$ and blocked in $3 \%(\mathrm{w} / \mathrm{v}) \mathrm{BSA} / \mathrm{PBS}$ for $1 \mathrm{~h}$ at RT. Immunolabeling was performed using a mouse anti-human monoclonal antibody against collagen type II (Col II; Chemicon, Temecula, CA, USA; dilution 1:5), a rabbit anti-human polyclonal antibody against Sox-9 (Milipore S.A.S, Molsheim, France; dilution 1:10) and a mouse anti-human monoclonal antibody against Aggrecan (ACAN; clone BC-3, Thermo Scientific, Waltham, MA, USA; dilution 1:5) as primary antibodies, prepared in $1 \%(\mathrm{w} / \mathrm{v}) \mathrm{BSA} / 0.2 \%(\mathrm{v} / \mathrm{v})$

Table 1

Primers list for the studied genes.

\begin{tabular}{lll}
\hline \multirow{2}{*}{ Gene } & Sequences & \\
\cline { 2 - 3 } & Forward $\left(5^{\prime}-3^{\prime}\right)$ & Reverse $\left(5^{\prime}-3^{\prime}\right)$ \\
\hline GADPH & TGCACCACCAACTGCTTAGC & GGCATGGACTGTGGTCATGAG \\
Col II & GACAATCTGGCTCCCAAC & ACAGTCTTGCCCCACTTAC \\
Sox-9 & TACGACTACACCGACCACCA & TTAGGATCATCTCGGCCATC \\
ACAN & TGAGTCCTCAAGCCTCCTGT & TGGTCTGCAGCAGTTGATTC \\
\hline
\end{tabular}

Triton X-100 in PBS solution and incubated overnight at $4{ }^{\circ} \mathrm{C}$. The sections were incubated for $2 \mathrm{~h}$ at RT with the respective secondary fluorochrome-conjugated antibodies, anti-rabbit/mouse IgG (Invitrogen, Life Technologies, California, USA; dilution 1:100), and with 4,6-Diamidino-2-phenyindole, dilactate (DAPI; Biotium, CA, USA; dilution 1:500) for 15 min for nuclei staining.

Histological and immunofluorescent sections were observed under transmitted and fluorescence microscopy (Col II and ACAN in green: ex/em 488/517; Sox-9 in red: ex/em 594/618; DAPI in blue: ex/em 358/461), respectively, using a transmitted and reflected light microscope. Images were acquired using the digital cameras AxioCam MRc5 and MR3, as described in 2.6.3. Scaffolds without cells were used for staining control.

\subsubsection{Dynamic mechanical analysis}

A dynamic mechanical analysis (DMA) was performed on the HRP-crosslinked SF scaffolds and cell-seeded constructs after 28 days of culture in chondrogenic differentiation medium, using a TRI-TEC8000B dynamic mechanical analyzer (Triton Technology, Lincolnshire, UK) in the compressive mode [27]. The measurements were carried out in the hydrated state at $37^{\circ} \mathrm{C}$. The diameter and height of the tested samples were 8 and $4 \mathrm{~mm}$, respectively. The samples were placed in a Teflon ${ }^{\circledR}$ reservoir and clamped in the DMA apparatus immersed in PBS solution. After equilibration at $37^{\circ} \mathrm{C}$, the DMA spectra were obtained in a frequency scan between 0.1 and $10 \mathrm{~Hz}$. A constant displacement amplitude of $50 \mu \mathrm{m}$ was maintained during the experiments. Both storage modulus $\left(E^{\prime}\right)$ and loss factor $(\tan \delta$ ) were obtained in the frequency range. Before the analysis, a small pre-load of $0.1 \mathrm{~N}$ was applied to each sample to ensure that the entire surface of the scaffold was in contact with the compression plates, being the distance between plates equal for all tested samples. At least four samples were used for analysis.

\subsection{Subcutaneous implantation}

In order to evaluate the in vivo biological response to the HRPcrosslinked SF scaffolds, the scaffolds were subcutaneously implanted in the back of twelve mice Hsd: ICR (CD-1) of 5 weeks old and average weight of 27-32 g at the time of implantation (Charles River Laboratories, Saint-Germain-sur-l'Arbresle, France). All animal procedures were based upon the "3Rs" policy (Replacement, Reduction and Refinement). The maintenance and use of animals were in accordance to the Ethics Committee of University of Minho, and approved by the Portuguese Licensing Authority (DGV-DSSPA).

Scaffolds of $8 \mathrm{~mm}$ in diameter and $4 \mathrm{~mm}$ in height were used for the subcutaneous implantation and sterilized by ethylene oxide. Each mouse was anesthetized by intraperitoneal injection of Domitor INJ $1 \mathrm{mg} / \mathrm{mL}$ (Medetomidine $1 \mathrm{mg} / \mathrm{Kg}$, Novavet, Braga, Portugal) and Imalgene 1000 INJ 100 mg-mL (Ketamine 75 mg/Kg, Novavet, Braga, Portugal). The hair of the mice was removed at the implantation area, followed by disinfection with iodine (Life Technologies, Carlsbad, CA, USA). In each mouse, two skin incisions ( $1 \mathrm{~cm}$ length) were made in the dorsal midline, close from the head $(\mathrm{CH})$ and far from the head (FH). The HRP-crosslinked SF scaffolds were then implanted subcutaneously at both right and left sides into the respective pockets, followed by skin suturing. Twelve specimens were implanted. The mice were euthanized 14 and 28 days postsurgery by injection of Eutasil $200 \mathrm{mg} / \mathrm{mL}$ (pentobarbital sodium, Novavet, Braga, Portugal) and the implanted materials were retrieved along with the surrounding tissue (Fig. 1d). The explants were fixed with $10 \%(\mathrm{v} / \mathrm{v})$ formalin for at least 5 days at RT and transferred to histological cassettes for paraffin-embedding. Samples were then serially sectioned using a microtome ( $3.5 \mu \mathrm{m}$ thick) 
and stained with hematoxylin \& eosin (H\&E). The histological sections were observed and images acquired as described in 2.6.8.

\subsection{Statistical analysis}

Statistical analysis was performed using the GraphPad Prism 5.0 software (GraphPad Software, La Jolla, CA, USA). First, a ShapiroWilk test was used to ascertain about the data normality. The compressive modulus results from static mechanical properties were analyzed by means of a Mann-Whitney test. A Kruskal-Wallis test, followed by Dunn's post-test were used to analyze the hASCs metabolic activity, proliferation, GAGs/DNA quantification and collagen content. The significance level was set to ${ }^{*} \mathrm{p}<0.05,{ }^{* *} \mathrm{p}<0.01$, $\mathrm{p}<0.001$

\section{Results}

\subsection{Morphology and microstructure characterization}

In this study, the detailed pore morphology at the HRPcrosslinked SF scaffolds surface (Fig. 2a and b) and cross-sections (Fig. $2 c$ and d) were analyzed by SEM. It was observed a similar macro-/micro-porous structure both at the scaffolds surface and interior, mainly with two types of pore size. From Fig. 2a and c, macro-pores with more than $500 \mu \mathrm{m}$ in size were observed. There were also micro-pores with less than $50 \mu \mathrm{m}$ distributed inside the thick macro-pore walls (Fig. 2b and d).

The qualitative and quantitative analysis of scaffolds architecture were investigated by micro-CT. Table 2 demonstrates that the HRP-crosslinked SF scaffolds presented a high porosity $(89.3 \pm 0.6 \%)$ and mean pore size $(361.4 \pm 20.3 \mu \mathrm{m})$, with interconnected pores $(95.9 \pm 0.8 \%)$ and thick pore walls $(51.7 \pm 2.9 \mu \mathrm{m})$. The two-dimensional (2D) horizontal cross-section (Fig. 2e) and $3 \mathrm{D}$ reconstruction of top and side views (Fig. $2 \mathrm{f}$ and $\mathrm{g}$ ) also showed the interconnectivity and porous structure of scaffolds, confirming the SEM observations. The micro-CT morphometric analysis revealed a widespread pore size and wall thickness distribution ranging from $8.79 \mu \mathrm{m}$ to 747.32 and $114.30 \mu \mathrm{m}$ within the scaffolds, respectively (Fig. $2 \mathrm{~h}$ and $\mathrm{i}$ ).

\subsection{Chemical structure and protein conformation}

The SF conformation and chemical composition in the scaffolds were analyzed by ATR-FTIR (Fig. 3). The FTIR spectra showed two
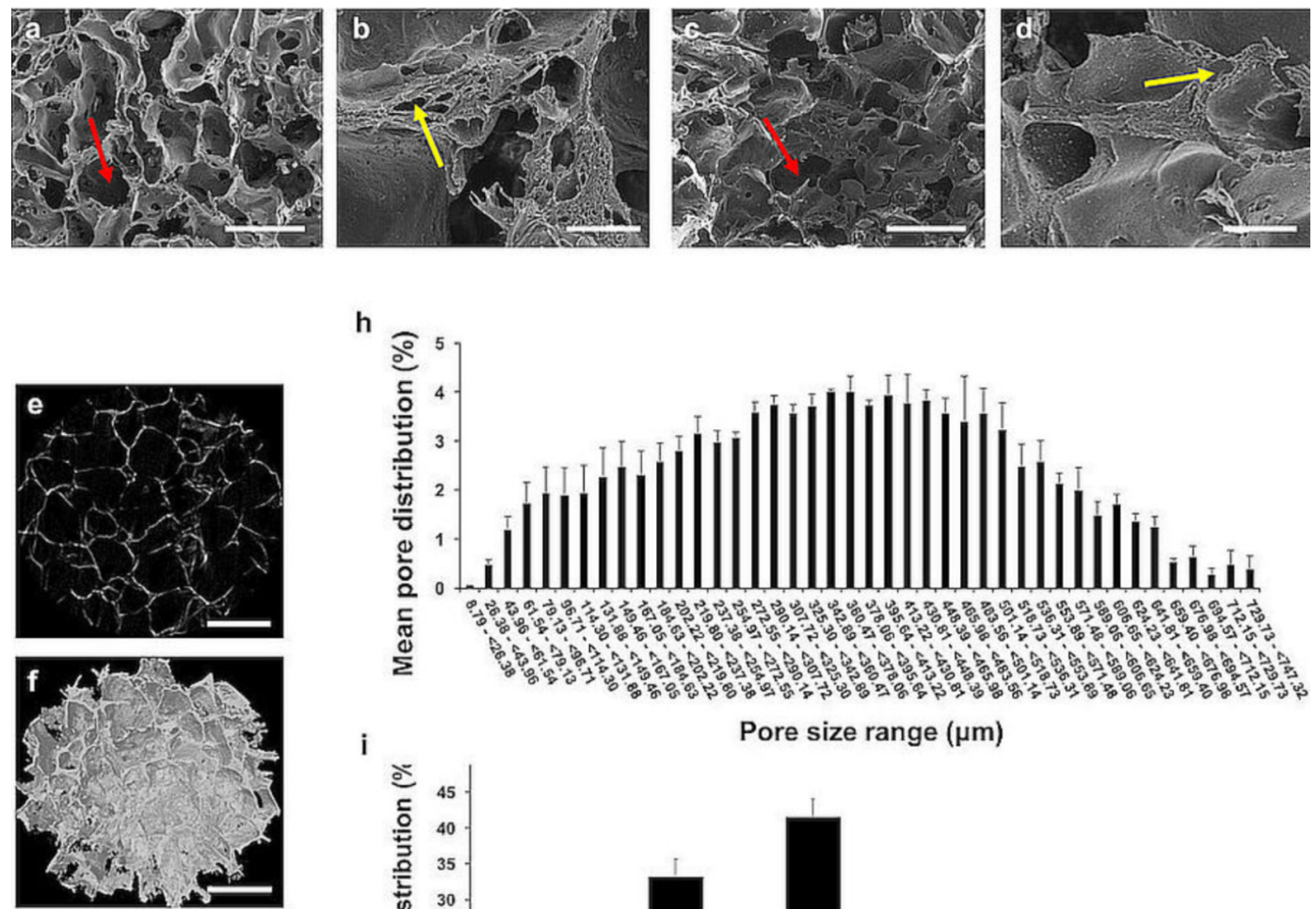

i

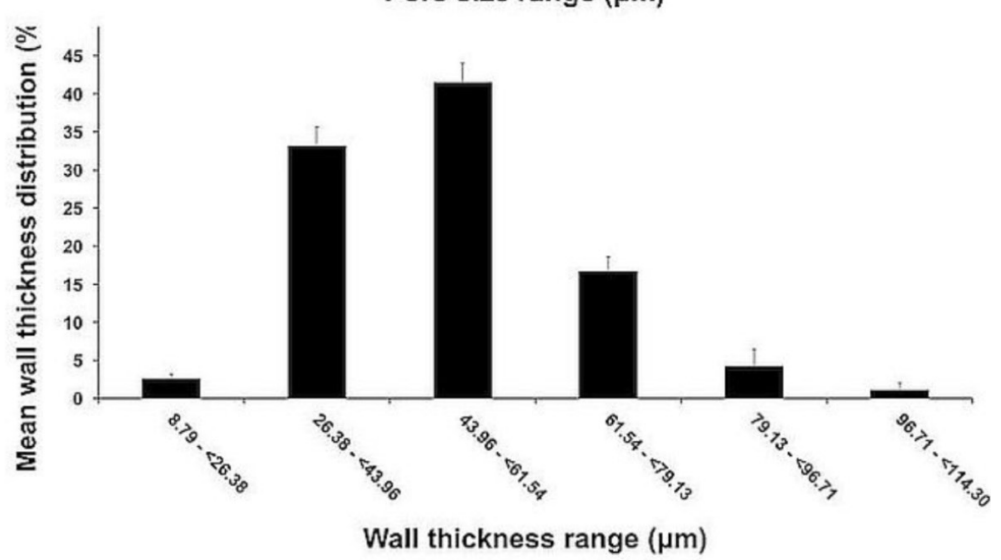

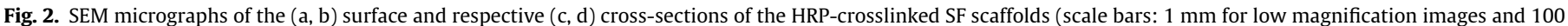

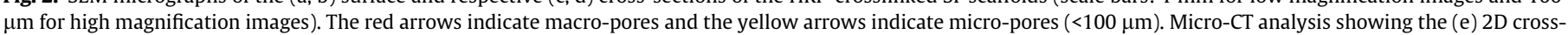

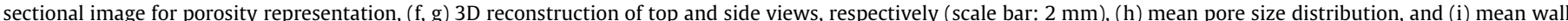

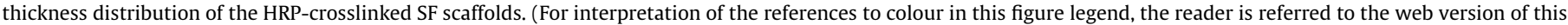
article.) 
Table 2

Microstructure of the HRP-crosslinked SF scaffolds determined from the micro-CT data.

\begin{tabular}{|c|c|c|c|c|}
\hline & Mean porosity (\%) & Mean pore size $(\mu \mathrm{m})$ & Mean interconnectivity (\%) & Mean wall thickness $(\mu \mathrm{m})$ \\
\hline HRP-crosslinked SF scaffold & $89.3 \pm 0.6$ & $361.4 \pm 20.3$ & $95.9 \pm 0.8$ & $51.7 \pm 2.9$ \\
\hline
\end{tabular}

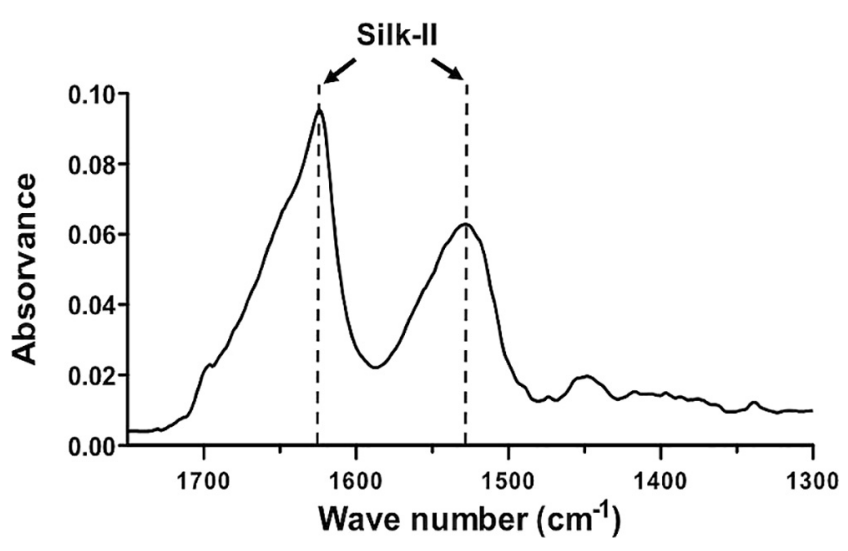

Fig. 3. ATR-FTIR spectra of the HRP-crosslinked SF scaffolds.

strong absorbance peaks at 1624 and $1528 \mathrm{~cm}^{-1}$. These two absorbance peaks are in the range assigned to amide-I and amide-II, respectively, and can be attributed to the $\beta$-sheet conformation of SF. A very small and hardly pronounced shoulder peak corresponding to the amorphous or silk-I conformation was detected at $\sim 1650 \mathrm{~cm}^{-1}$ [39].

\subsection{Mechanical properties, swelling ratio and degradation profile}

As shown in Fig. 4a, the static compressive modulus of the dried HRP-crosslinked SF scaffolds was $2.97 \pm 0.90 \mathrm{MPa}$, significantly higher than the wet state modulus of $0.11 \pm 0.07 \mathrm{MPa}$ $\left({ }^{* * *} \mathrm{p}<0.01\right)$. The representative stress-strain plot (Fig. $\left.4 \mathrm{~b}\right)$ shows the same behavior for the compressive strength of scaffolds in dry $(0.54 \pm 0.05 \mathrm{MPa})$ and wet $(0.03 \pm 0.006 \mathrm{MPa})$ conditions.

Fig. $4 \mathrm{c}$ shows that the swelling ratio of the HRP-crosslinked SF scaffolds reached an equilibrium after $6 \mathrm{~h}$ of immersion in PBS ( $\sim 585 \%)$ solution, maintaining their weights up to 30 days. In this study, the enzymatic degradation of the HRP-crosslinked SF scaffolds was analyzed by using protease XIV [40]. It was observed that the degradation rate of scaffolds was faster in the presence of protease XIV at both tested concentrations ( $3.5 \mathrm{U} / \mathrm{mL}$ and $0.2 \mathrm{U} / \mathrm{mL}$ ), as compared to the control condition in which scaffolds maintained their original weights after soaking in PBS solution for 30 days. In the first $12 \mathrm{~h}$, the degradation rate of scaffolds immersed in protease XIV at $3.5 \mathrm{U} / \mathrm{mL}$ was higher than $50 \%$, as compared to the $\sim 20 \%$ weight loss in protease XIV at $0.2 \mathrm{U} / \mathrm{mL}$. After 14 days of degradation in protease XIV at $3.5 \mathrm{U} / \mathrm{mL}$, scaffolds reached $100 \%$ weight loss, while the scaffolds immersed in protease XIV at $0.2 \mathrm{U} / \mathrm{mL}$ presented $\sim 60 \%$ weight loss only after 30 days of degradation.

\subsection{In vitro characterization of the HRP-crosslinked SF scaffolds}

Prior differentiation, hASCs were characterized for the expression of MSCs surface markers, CD105, CD90, CD73, CD45 and CD34. The cells showed a mesenchymal-like phenotype, exhibiting the typical panel of markers: $\mathrm{CD}_{105^{+}} / \mathrm{CD}^{+} 3^{+} / \mathrm{CD}^{+} 0^{+} / \mathrm{CD} 45^{-} / \mathrm{CD} 34^{-}$ [37] (Supplementary Fig. 1). a

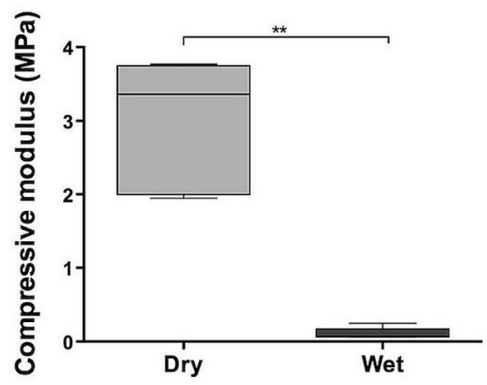

C

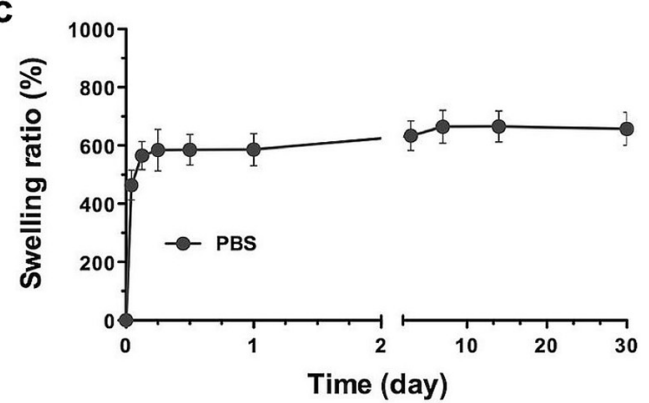

b

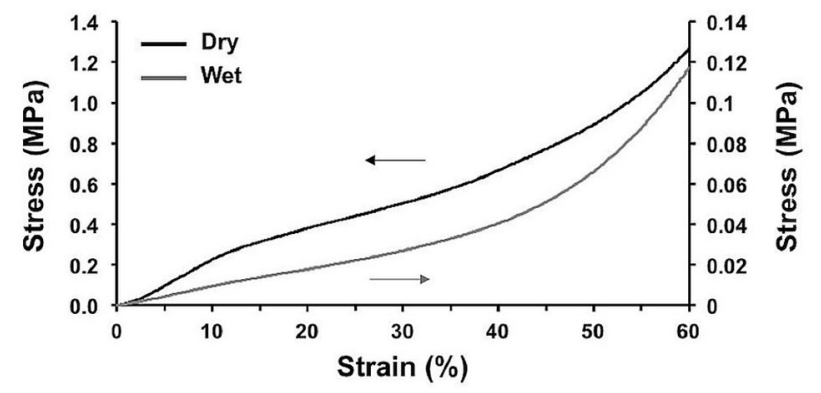

d

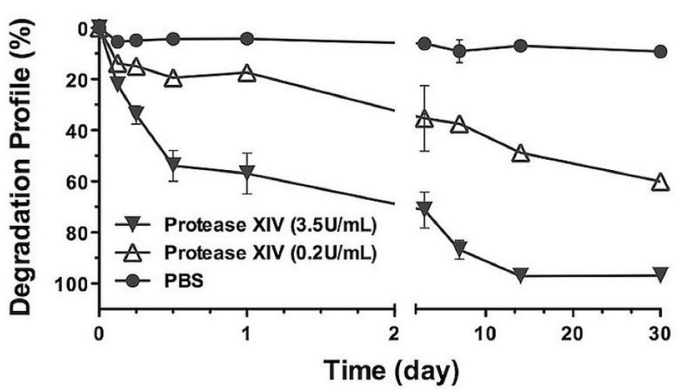

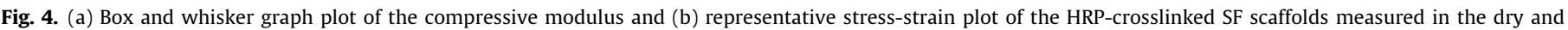

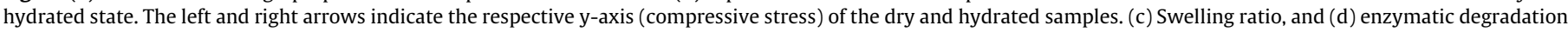
profile of the HRP-crosslinked SF scaffolds for a period of 30 days (\% weight). 


\subsubsection{Metabolic activity and cell viability}

The Alamar blue assay was performed to evaluate the metabolic activity of cells up to 28 days of culturing (Fig. 5a). In basal culture conditions, cells presented a significant increase of metabolic activity over the 28 days of culture $\left({ }^{*} p<0.05\right.$ from day 7 to day 14 ; ${ }^{* *} \mathrm{p}<0.01$ from day 1 to day $21 ;{ }^{* * *} \mathrm{p}<0.001$ from day 7 to day 21 and from day $1,7,14$ to day 28). Concerning the cultures in chondrogenic differentiation medium, stable metabolic activity levels were observed from day 1 to day 28. However, at day 1 and day 7 , cells presented a significantly higher metabolic activity as compared to that detected in basal culture conditions $\left(a^{* * *} p<0.001\right)$. From the live/dead staining, it was found that cells adhered to the highly porous scaffolds and were alive over the 28 days of culture in chondrogenic and basal conditions (Fig. 5b). From day 7, a dense cell layer spreading over the HRP-crosslinked SF constructs was observed in both culture media. Nevertheless, at day 21 and day 28 the presence of self-aggregated cells clusters was observed in chondrogenic culture conditions, replacing the dispersive and elongated morphology of cells observed in basal medium at the same culture periods.

\subsubsection{Biochemical characterization}

Cell proliferation was screened by DNA content analysis (Fig. 6a). It was observed that no significant differences in cell proliferation were observed from day 1 to day 28 for cells cultured in chondrogenic differentiation medium. The DNA content significantly increased in basal culture conditions from day 1 to day 28 $\left({ }^{*} \mathrm{p}<0.05\right.$ : from day 7 to day $21 ;{ }^{* *} \mathrm{p}<0.01$ : from day 1 to day 28 ; $\mathrm{p}<0.001$ : from day 7 to day 28) presenting at day 21 $\left(a^{* *} \mathrm{p}<0.01\right)$ and day $28\left(b^{* * *} \mathrm{p}<0.001\right)$ significantly higher DNA content than in chondrogenic culture conditions.
The GAGs production from the cells seeded on the HRPcrosslinked SF scaffolds was normalized by their respective DNA content (Fig. 6b). It was found a significant increase of GAG/DNA ratio after 7 days of culture in chondrogenic differentiation medium, in comparison to day $1\left({ }^{* *} \mathrm{p}<0.01\right)$. No significant differences were observed at day 14 . However, after 21 days and 28 days in chondrogenic culture conditions the GAG/DNA ratio significantly increased as compared to day $1\left({ }^{* * *} \mathrm{p}<0.001\right)$. In basal culture medium, only a residual GAG/DNA ratio was determined and no significant differences were observed over the 28 days of culture. In fact, the GAG/DNA ratio determined on the HRP-crosslinked SF constructs cultured in chondrogenic differentiation medium was significantly higher at day $21 \quad\left(\mathrm{~b}^{* * *} \mathrm{p}<0.001\right)$ and day 28 $\left(\mathrm{a}^{* * *} \mathrm{p}<0.01\right)$, as compared to that obtained in basal culture conditions.

The quantification of the proteins that integrate the ECM was performed by Sirius red/Fast green staining kit, aiming at discriminate the collagen content on the HRP-crosslinked SF constructs. In Fig. 6c, a non-significant increase of the collagen content was determined after 21 days of culture in chondrogenic differentiation medium. For the HRP-crosslinked SF constructs cultured in basal conditions, a significant increase of the collagenous proteins was observed after 21 and 28 days of culture, as compared to day 1 $\left({ }^{*} \mathrm{p}<0.05\right)$, day $7\left({ }^{* *} \mathrm{p}<0.01\right)$ and day $14\left({ }^{* * *} \mathrm{p}<0.001\right)$. When comparing the chondrogenic and basal culture conditions at the same culture period, no significant differences were detected for the collagen deposition at the HRP-crosslinked SF constructs.

3.4.3. Chondrogenic-related markers expression, extracellular matrix composition and dynamic mechanical properties after culturing

The differentiation of hASCs seeded onto the HRP-crosslinked SF scaffolds was evaluated by quantifying the mRNA expression of the

a

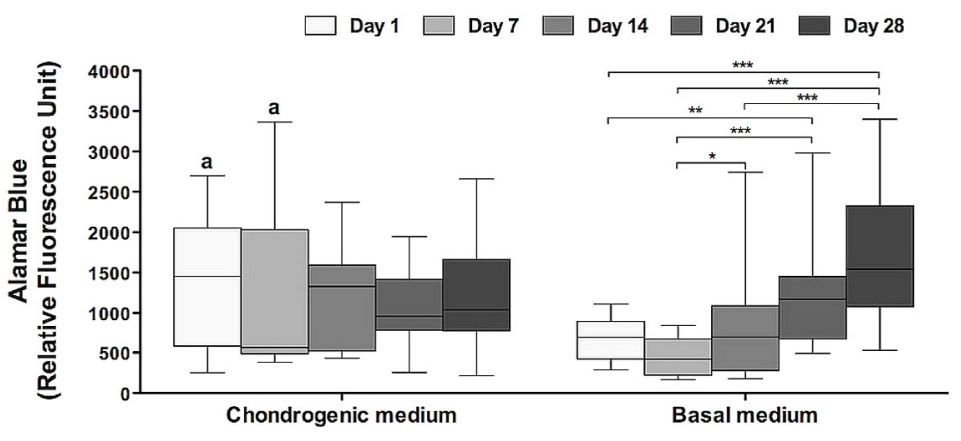

b
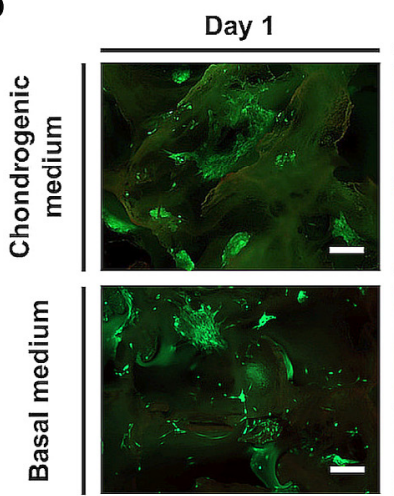

Day 7
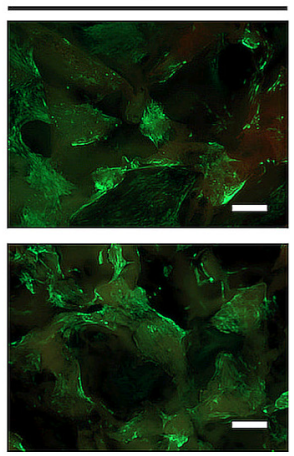

Day 14
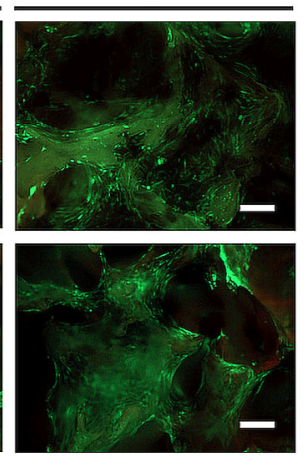

Day 21
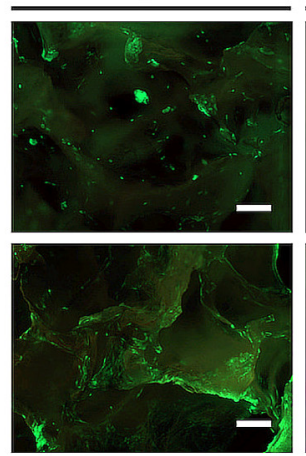

Day 28

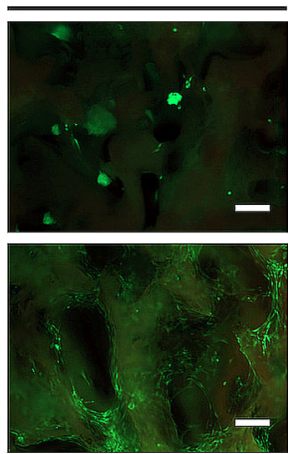

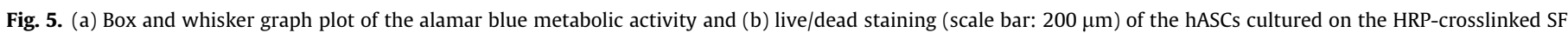

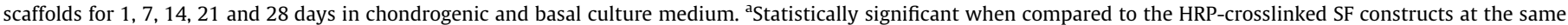

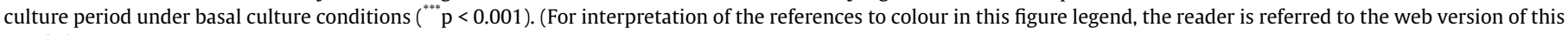
article.) 

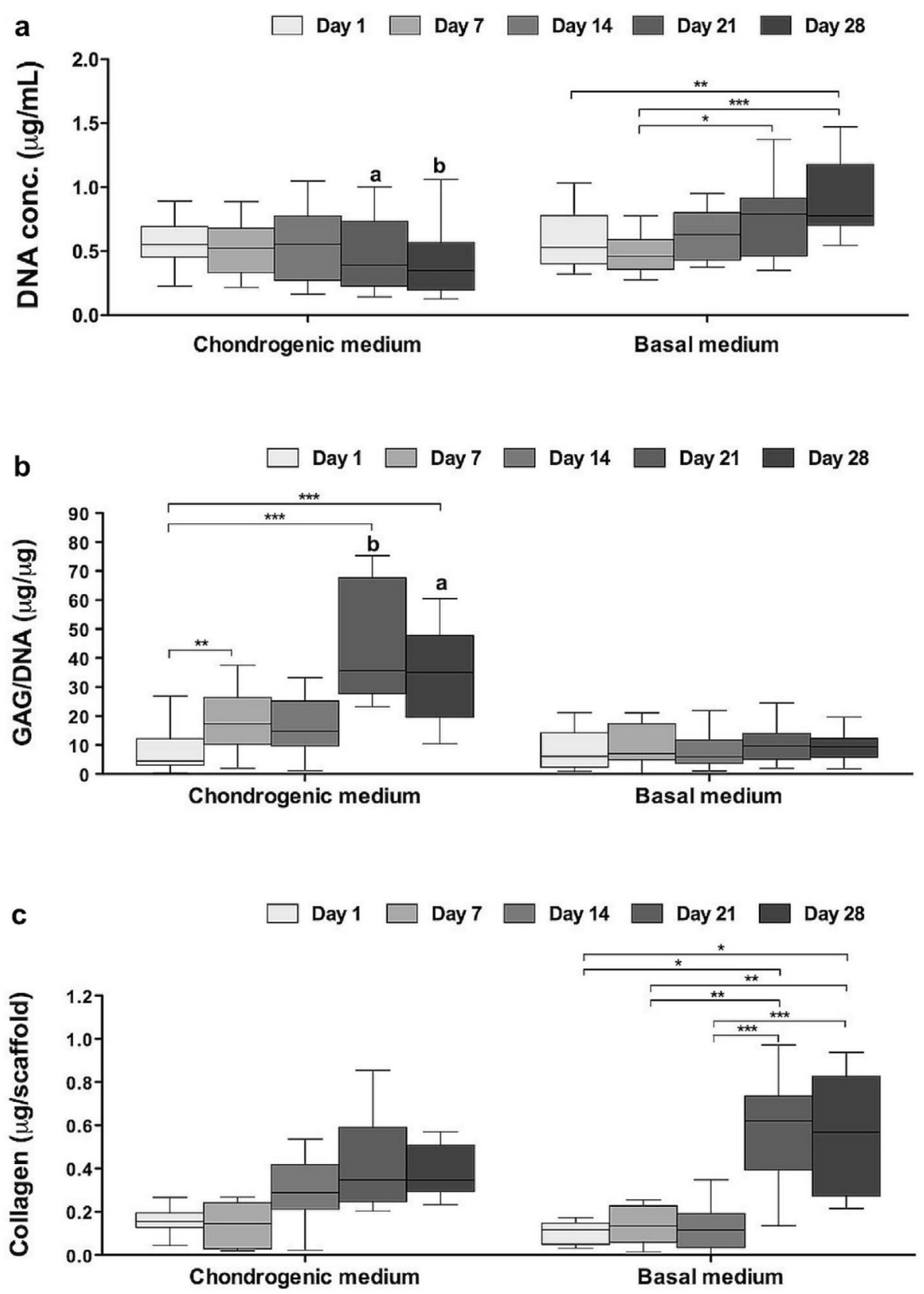

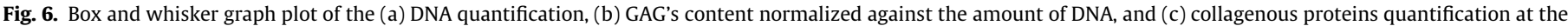

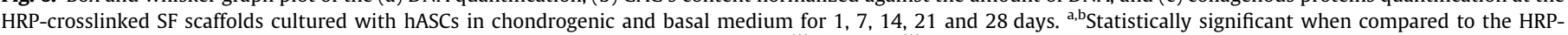
crosslinked SF constructs at the same culture period under basal culture conditions $\left(\mathrm{a}^{* * * *} \mathrm{p}<0.01, \mathrm{~b}^{* * *} \mathrm{p}<0.001\right)$.

chondrogenic-related markers, collagen type II (Col II), Sox-9 and Aggrecan (ACAN) (Fig. 7a). The mRNA transcripts evaluated after 28 days of culture in basal and chondrogenic differentiation medium, revealed the expression of all chondrogenic-related markers after culturing the HRP-crosslinked SF constructs in chondrogenic differentiation medium. In contrast, no mRNA expression was detected for the same markers in basal culture conditions.

Complementary, the immunodetection of Col II, Sox-9 and ACAN at the HRP-crosslinked SF constructs was also evaluated (Fig. 7b). The immunofluorescence images show an intense protein expression of all chondrogenic-related markers after 28 days of culture in chondrogenic differentiation medium. However, in basal culture conditions only a residual expression of Col II, Sox-9 and ACAN was detected.
The newly formed ECM at the HRP-crosslinked SF constructs was evaluated and sections stained to visualize the GAGs secretion and collagen deposition (Fig. 7c). After 28 days of culture, only the ECM formed on the HRP-crosslinked SF constructs cultured in chondrogenic differentiation medium was intensely stained for GAGs, as observed from the intense Alcian blue and positive staining for Safranin-O. Moreover, from the Masson's trichome and Sirius red staining's it was possible to observe a well pronounced collagen matrix deposited on the HRP-crosslinked SF constructs cultured both under chondrogenic and basal culture conditions.

The dynamic viscoelastic properties of the HRP-crosslinked SF scaffolds and constructs after 28 days of culture in chondrogenic differentiation medium were assessed by DMA (Fig. 7d), in order 
a

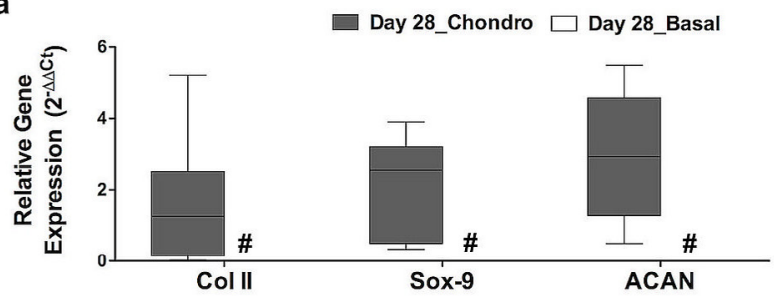

b
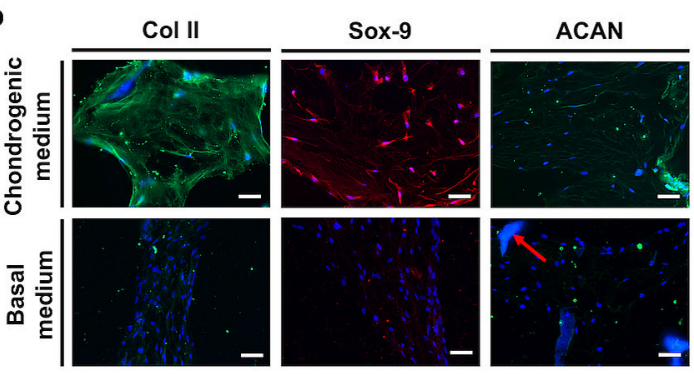

Control

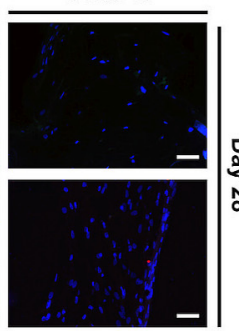

C

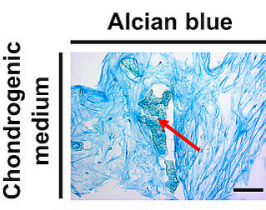

Safranin-O

Sirius red
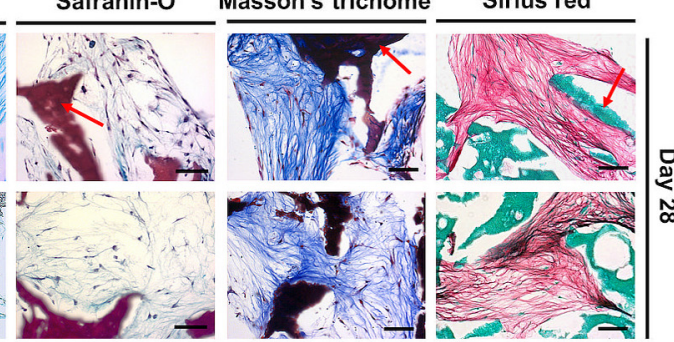

d

- HRP-crosslinked SF constructs

$\nabla$ HRP-crosslinked SF scaffolds
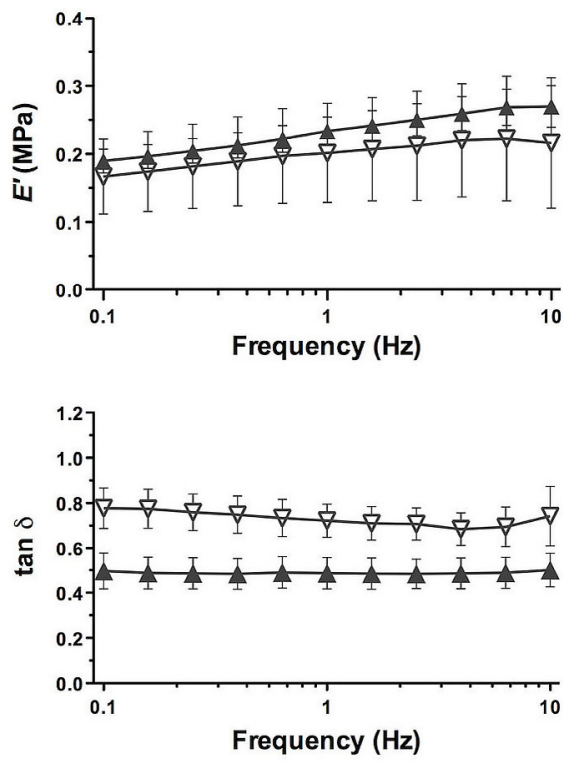

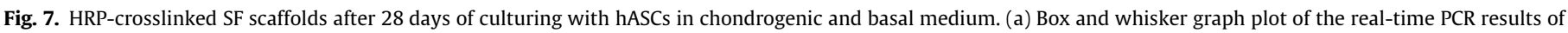

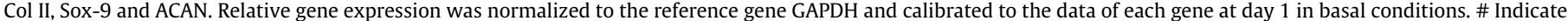

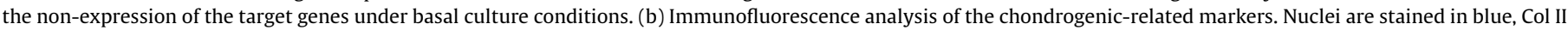

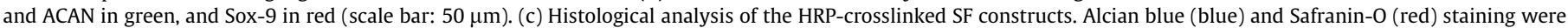

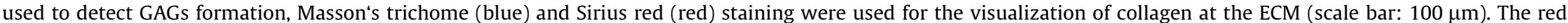

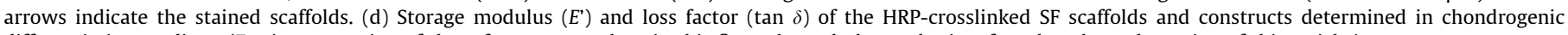
differentiation medium. (For interpretation of the references to colour in this figure legend, the reader is referred to the web version of this article.)

to evaluate and compare the dynamic mechanical behavior of scaffolds for cell-free and cell-based approaches in cartilage TE applications. It was observed that the storage modulus ( $\left.E^{\prime}\right)$ of the HRP-crosslinked SF scaffolds and constructs slightly increased from $0.17 \pm 0.06$ to $0.22 \pm 0.10 \mathrm{MPa}$ and $0.19 \pm 0.02$ to $0.27 \pm 0.03$ $\mathrm{MPa}$, respectively, as the frequency increased from 0.1 to $10 \mathrm{~Hz}$. In the frequency range tested the $E^{\prime}$ values of the HRPcrosslinked SF constructs were higher than those obtained for the scaffolds. Comparing the loss factor $(\tan \delta$ ) results, it was found that both groups presented stable and high $\tan \delta$ values for the tested frequencies. Nevertheless, it was found that the HRPcrosslinked SF scaffolds presented higher $\tan \delta$ values than constructs.

\subsection{Subcutaneous implantation of the HRP-crosslinked SF scaffolds}

The in vivo compatibility of the HRP-crosslinked SF scaffolds was assessed by subcutaneous implantation in a mice model (Fig. 8). The macroscopic images of the explants showed that scaffolds were still integrated in the subcutaneous tissue after 14 (Fig. 8a) and 28 (Fig. 8b) days of implantation, maintaining their shape and integrity over the implantation periods. From the low magnification H\&E staining images (Fig. 8c and d), it was observed that a thick layer of connective tissue adhered on the entire surface of the scaffolds and deeply infiltrated into the porous structures. The high magnification H\&E staining images (Fig. 8e-h) allowed confirming the presence of connective tissue filling the inner pores of the scaffolds. The absence of edema or signs of neutrophils after the implantation periods, revealed that no acute inflammation was induced by the HRP-crosslinked SF scaffolds.

\section{Discussion}

In natural cartilage tissue, the ECM provides a complex environment of physical, chemical and mechanical cues that directly affect and guide cell behavior. In the recent years, SF has demonstrated to be a robust natural material widely explored for bone [41], cartilage [27] and osteochondral [42] TE. Its processing versatility allowed to extend the use of SF for TE applications of ligament [43], blood vessels [44] or skin [45]. Several methods have been individually applied to prepare silk scaffolds [41,44,46], frequently with the limitation of poorly mimicking the structural and mechanical complexity of tissues like cartilage. The salt-leaching method is widely used due to the effective processing and generated controlled porosity in a wide range of pore sizes [47]. Moreover, the behavior of SF in the presence of $\mathrm{NaCl}$ is affected by the salt ions extracting the water that usually coat the hydrophobic domains in the $\beta$-sheet structure of the protein. This would promote chain-chain interactions resulting in protein folding and $\beta$-sheet formation $[48,49]$. Salt-leached silk scaffolds generally present high stiffness due to the presence of silk II ( $\beta$-sheet conformation) formation. For example, Kim et al. [48] developed aqueous-derived SF scaffolds prepared using salt-leaching and freeze-drying methodologies, showing that scaffolds had controlled pore size, interconnectivity and adjustable mechanical properties. In a different study, Nazarov et al. [46] individually 

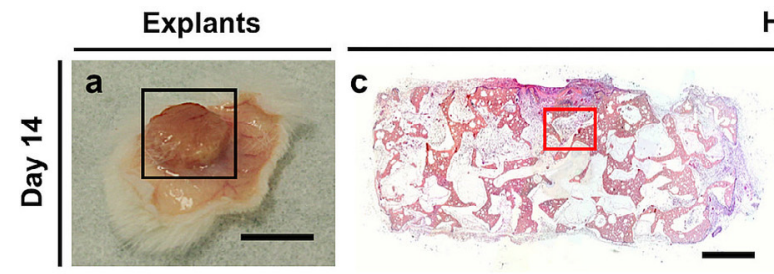

$H \& E$
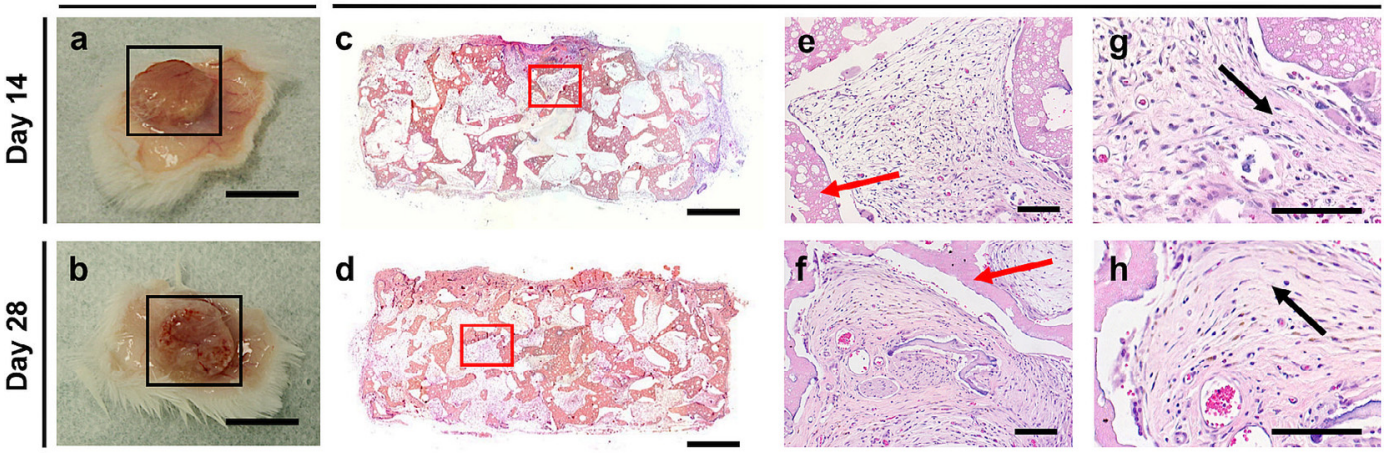

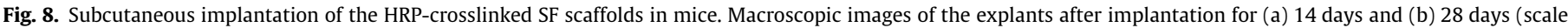

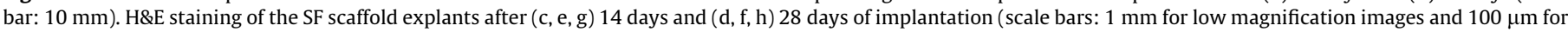

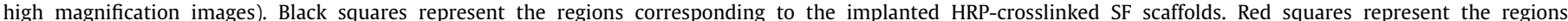

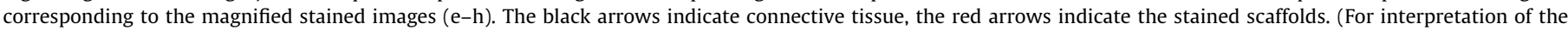
references to colour in this figure legend, the reader is referred to the web version of this article.)

applied the salt-leaching and freeze-drying techniques to form porous 3D silk biomaterials, stating that different matrices can be produced according to the processing method and conditions. Moreover, these authors also showed that to induce $\beta$-sheet structure and water insolubility after lyophilization, a methanol treatment was induced to the aqueous SF before freeze-drying processing. In our study, the crystallinity of the HRP-crosslinked SF solution was ensured with the salt-leaching processing, avoiding the return to an amorphous and gel state after re-hydration of the freeze-dried scaffolds, as confirmed by FTIR analysis (Fig. 3). According to different authors [13,14], scaffolds designed for cartilage TE should include optimal structural and mechanical properties, controlled degradability rate and good adaptability to the defect site, showing that highly porous SF scaffolds developed by salt-leaching and freeze-drying methodologies can fit to these criteria. In a previous study by our group [27], salt-leached and freeze-dried SF scaffolds prepared in a range of highconcentration aqueous SF solutions, showed stable degradability and improved mechanical properties for meniscus and cartilage regeneration. Nevertheless, to fully recreate the microenvironment found in cartilage tissue, there is a need to better control the stiffness of the salt-leached SF scaffolds. Until now, SF hydrogels have been seen as potential matrices for cartilage applications presenting a biomimetic aqueous matrix responsible for its resilient and elastic behavior [12]. However, these matrices can also be less stable for cell culture applications, raising the hypothesis of creating novel SF biomaterials that match the stiffness of soft SF hydrogels with the porosity and mechanical stability of salt-leached SF scaffolds. In a recent study, agarose/SF blended hydrogels were structured by freeze-drying methodology to create porous structure of hydrogels, showing the typical elastic behavior of blended hydrogels but at the same time a reinforcement and stability induced by the microporous structures [7]. Moreover, the cellular studies demonstrated that chondrocytes cultured in the structures with larger pores induced higher cartilage-specific ECM production. To achieve this effect, the herein proposed scaffolds resulted from a combination of enzymatically crosslinked SF hydrogels with salt-leaching and freeze-drying technologies to create a highly porous, stable and yet stiff hydrogel-based system for specific cartilage TE applications. As demonstrated by the SEM (Fig. 2a-d) and micro-CT (Fig. 2e-i and Table 2) analysis not only a high porosity was observed within the HRP-crosslinked SF scaffolds, as different pores sizes were achieved. Considering the wide size range of the sodium chloride particles used for salt-leaching processing, it was expected that the pore size of the scaffolds would not be homoge- neous, even presenting a homogeneous distribution along the scaffolds (Fig. 2h). Moreover, the thick pore walls $(51.7 \pm 2.9 \mu \mathrm{m})$ induced by the highly concentrated aqueous SF solution, also demonstrated small pores $(<50 \mu \mathrm{m})$ distributed inside the structures (Fig. 2b and d) that can result from the recrystallization of the dissolved salt particles (leaching process) combined with the freeze-drying process [27]. This wide range of pore size is of great interest for cartilage TE, especially due to the lack of selfremodeling capacity of cartilage tissue that naturally present small pores ( 2.5 to $6.5 \mathrm{~nm}$ ). Pores with sizes inferior to $50 \mu \mathrm{m}$ are recommended to improve cell adhesion and seeding efficiency, whereas larger and interconnected pores improve cellularity and ECM ingrowth for faster constructs development [50]. As reported by Vikingsson et al. [51] different pore sizes and wall thickness within the micro-architecture can directly affect the mechanical properties and degradation profile of scaffolds. In a previous study, Han et al. [8] observed that the compressive strength of salt-leached/ freeze-dried SF scaffolds produced with aqueous SF solutions of 4-5 wt\%, decreased from 1.6 to $1.09 \mathrm{MPa}$ as the pore size increased (90-180 $\mu \mathrm{m}$ to $355-425 \mu \mathrm{m}$ ). This was an expected result, since a lower macro-porosity induce the formation of denser structures that can withstand higher loads. As observed in our study, Kim et al. [48] also showed a compressive modulus of $3.33 \pm 0.5 \mathrm{MPa}$ and a compressive strength of $0.32 \pm 0.01 \mathrm{MPa}$ on dried saltleached/freeze-dried SF scaffolds, produced with high concentration SF solution (10 wt\%) and bigger particle sizes (1,000-1,180 $\mu \mathrm{m})$. An important role is also attributed to the $\beta$-sheet content of SF scaffolds. In the present study, superior $\beta$-sheet content and less amount of amorphous conformation was detected by FTIR (Fig. 3), which may have influenced the mechanical properties of the produced scaffolds. In a different study performed by Yao et al. [52], the compressive modulus and compressive strength of salt-leached SF scaffolds was higher on samples with higher silk II ( $\beta$-sheet crystal) formation. Yan et al. [16], also showed that the compressive modulus of the HRP-crosslinked SF hydrogels with $\beta$-sheet conformation (1.12 MPa), was significantly higher than that of random coil hydrogels $(0.022 \mathrm{MPa})$. When compared to the compressive modulus of the crystalline HRP-crosslinked SF scaffolds, both presented similar compressive modulus to the native human articular cartilage tissue (ranging from 1.16 to 7.75 MPa in deep, from the articular surface) [53].

It is well-established that implantable scaffold materials should be able to absorb the nutrients and metabolites from the adjacent tissue. Moreover, the swelling process is also necessary to press-fit the construct at the implantation site, improving the fluidic 
exchanges [40]. At the same time, the constructs should be able to restore the biomechanical function and maintain a controllable degradation during the stages of manipulation and thereafter.

Biomaterials degradation in the living environment is a complex phenomenon that involves several synergistic pathways, either enzymatic or chemical [54]. Thus, the in vitro degradation should provide a realistic clue of the functional interaction between the construct and the biological environment. Protease XIV have been used for in vitro degradation studies involving SF structures [42,54]. This enzyme has no specific activity towards the chemical structure and amino acid sequence of SF, which makes it more aggressive to better mimic the in vivo conditions that simultaneously involve the activity of several enzymes (chymotrypsin, collagenase, etc.) [55]. In the present study, we have proposed HRP-crosslinked SF scaffolds with large swelling capacity and able to maintain a swelling stability and structural integrity over 30 days (Fig. 4c). In our previous study, we also found that the HRP-mediated SF hydrogels are $\mathrm{pH}$ responsive maintaining their original weights and structure at physiological conditions [25]. In a different study, Yan et al. [16] observed that random coil SF hydrogels presented higher swelling capacity than the crystalline SF hydrogels, due to the aggregated SF molecules formed with the $\beta$-sheet induction that make the hydrogels more hydrophobic. Moreover, these strong hydrophobic interactions may have rendering the protease XIV more inaccessible for proteolytic cleavage, affecting the degradation of the crystalline SF hydrogels, which presented only $5 \%$ weight loss after $12 \mathrm{~h}$ in protease XIV solution $(0.2 \mathrm{U} / \mathrm{mL})$, as compared to the faster degradation of the random coil SF hydrogels. Our results showed a greater impact on the HRP-crosslinked SF scaffolds degradation at the highest tested concentration of protease XIV $(3.5 \mathrm{U} / \mathrm{mL})$, reaching $100 \%$ weight loss after 14 days in degradation solution. However, the degradation profile tested with $0.2 \mathrm{U} / \mathrm{mL}$ of enzyme, showed a $19 \%$ weight loss within $12 \mathrm{~h}$, which can be explained by the high porosity and mean pore size (Table 2) attributed to the salt-leaching processing [56]. In a previous study by our group [42], it was observed that SF scaffolds derived from highly concentrated aqueous SF solution (16 wt\%) and processed through saltleaching (500-1,000 $\mu \mathrm{m}$ sodium chloride particles size) and freeze-drying technologies, presented a much faster degradation rate as compared to the HRP-crosslinked SF scaffolds. In this case, the covalent bonds established between the tyrosine groups in the enzymatic crosslinking process can difficult the proteolytic cleavage induced by the protease XIV, making the degradation process slower and more controlled $[16,25]$. These results correlate well with the literature, stating that the degradation properties of scaffolds should be tailored to match the target tissue regeneration [1]. Considering the limited self-remodeling capacity of cartilage tissue, a slower degradation profile of the HRP-crosslinked SF scaffolds can be appropriate for cartilage scaffolding strategies.

Successful cell-based TE strategies for the repair of critical-sized cartilage defects also require a safe and easily accessible cell source for in vitro chondrogenesis and eventually functional integration in vivo [57]. Human ASCs have demonstrated a significant potential for chondrogenic differentiation, using different scaffolding strategies as platforms $[33,58]$. Nevertheless, as reported in previous studies, a wide range of scaffolds pore size can induce different cell behaviors $[7,8]$. In this study, we were able to observe the effects of scaffolds micro-porosity on a good cell adhesion and spreading at the surface, as well as, cells infiltrating the interconnected large pores to colonize the scaffolds interior (Fig. 5b). In chondrogenic differentiation medium it was possible to observe that the porous 3D environment induced cell clusters formation, typically found during cartilage development [59]. Han et al. [8] also observed that salt-leached SF scaffolds produced with different pore size had distinct influence on chondrocytes behavior. Scaffolds with smaller pore size improved cell adhesion and presented the highest levels of GAGs and collagen content, whereas the scaffolds with higher pore size provided the best environment for cell proliferation. In our study, cell proliferation was stable until day 14 on both chondrogenic and basal culture conditions, however, at day 21 and day 28 significantly higher DNA values were detected in basal culture conditions, as compared to HRP-crosslinked SF constructs in chondrogenic differentiation medium (Fig. 6a). These results corroborate the metabolic activity (Fig. 5a) and live/dead (Fig. 5b) observations, suggesting the differentiation of hASCs in the HRPcrosslinked SF constructs under chondrogenic culture conditions. Confirming these observations, the amount of released GAGs by the chondrogenic-induced hASCs at day 21 and day 28 was significantly higher than that in basal culture conditions (Fig. 6b). Moreover, the quantification of collagen content showed the same tendency for an increase of protein deposition until day 21 (Fig. 6c). Previous studies, showed the positive influence of the porous-like structure of SF scaffolds on hASCs chondrogenic differentiation and cartilage-specific ECM production [33,34]. For example, Kim et al. [33] observed that chondrogenic-induced hASCs were able to produce higher GAGs and collagen content on saltleached SF scaffolds, as compared to those in the conventional micromass pellet culture system [60].

In vitro chondrogenic differentiation is characterized by the genotypic (Fig. 7a) and phenotypic (Fig. 7b) expression of cartilage-specific markers. Col II is the most common type of collagen at the hyaline cartilage, and together with ACAN are two of the most important components of cartilage ECM. Sox-9 is a key transcription factor in chondrogenesis able to directly regulate the expression of Col II and ACAN [14]. In this study, the analysis of Col II, Sox-9 and ACAN showed their expression after 28 days of culturing in chondrogenic differentiation medium, which may be indicative of hASCs chondrogenic differentiation on the HRPcrosslinked SF constructs. This behavior has been reported in other studies $[33,34]$ and is also in agreement to the GAGs (Fig. 6b) and collagen (Fig. 6c) quantification results. Moreover, the nonexpression of these markers under basal culture conditions also corroborates to this hypothesis.

The GAGs secreted by the chondrogenic-induced hASCs were also confirmed by histological analysis (Fig. 7c). However, the collagen deposition was shown at similar levels on both chondrogenic and basal culture conditions, which is not in agreement to the results obtained for Col II expression (Fig. 7a and b). In fact, it is well known that other type of collagen is associated with the ECM of undifferentiated cells [61]. Therefore, the total collagen quantified (Fig. 6c) and visualized (Fig. 7c) at the ECM of the HRP-crosslinked SF constructs cultured in basal medium may not correspond to the Col II observed in chondrogenic culture conditions. After 28 days of culture in chondrogenic differentiation medium, a slight increase of the storage modulus was determined on the HRP-crosslinked SF constructs, as compared to the nonseeded scaffolds (Fig. 7d), which can be explained by the presence of the newly formed ECM. Nevertheless, there were no statistical differences in respect to the mechanical properties with or without chondrogenic cell culture, which represents the ability of scaffolds to maintain their mechanical performance during tissue regeneration [27]. Considering that a healthy articular cartilage sustains physiological stresses that can range from 1 to $10 \mathrm{MPa}$, the proposed HRP-crosslinked SF constructs can be suggested as temporary cartilage tissue substitutes [62]. The loss factor results (Fig. 7d), also confirmed the viscoelastic nature of both HRPcrosslinked SF scaffolds (ranging from 0.8 to 0.74 ) and constructs (ranging from 0.49 to 0.50 ), which means that the proposed structures can completely recover from high physical loads, which is an important concern considering that articular cartilage of joints are highly stimulated mechanically, from the daily walking to the 
active loading sports [2]. Moreover, these results suggest that these structures can be easily adjusted to fulfill cartilage defects and facilitate surgical interventions.

The compatibility between the HRP-crosslinked SF scaffolds and in vivo tissues evaluated by subcutaneous implantation, showed good scaffolds integrity and dimensional stability during the implantation periods (Fig. 8a and b). The crystalline structure of scaffolds, confirmed by FTIR (Fig. 3) analysis, may have influenced the obtained results. Moreover, the stable degradation profile of the HRP-crosslinked SF scaffolds (Fig. 4d), also corroborates to this hypothesis. In a previous study by our group [42], the in vivo compatibility of bilayered SF/SF-nanoCaP scaffolds was also tested by subcutaneous implantation up to 28 days, showing that the crystalline structure of SF allied to the suitable mechanical properties of scaffolds maintained their stability and shapes during the implantation period. A thick layer of connective tissue was observed surrounding and deeply infiltrating the HRP-crosslinked SF scaffolds (Fig. 8e-h). The high porosity and interconnectivity revealed by SEM (Fig. 2a-d) and micro-CT (Fig. 2e-i and Table 2) analysis may have contributed to these results $[41,42]$. For example, Yan et al. [42] have also demonstrated the positive influence of highly porous structures to support tissue ingrowth on bilayered SF/SF-nanoCaP scaffolds. The surface chemistry of SF is known for providing good cell behavior and biocompatibility in vivo [41], showing that the in vitro and in vivo performance of the HRPcrosslinked SF scaffolds may not be only related to the architecture and morphological features of constructs, but also to the physical and chemical properties of SF [63]. The versatility of the present combined technologies, and the possibility of incorporating inorganic materials can encourage extending their application in different regenerative strategies, in particular, for developing monolithic and hierarchical structures for osteochondral regeneration.

A number of limitations of the present study offer opportunities for additional research. First, a control condition using macro-/ micro-porous SF scaffolds produced with pure SF solution (16 wt $\%)$ would allow to be compared with the proposed HRPcrosslinked SF scaffolds. Also, this study focused on the effect of using established concentrations of $\mathrm{SF} / \mathrm{HRP} / \mathrm{H}_{2} \mathrm{O}_{2}$. The concentrations could be changed (in proportion) in order to evaluate the effects over scaffolds mechanical properties and degradation profile [25]. Additionally, testing a range of macro-porosity induced by the salt-leaching processing would be of great interest. Finally, an in vivo study performed to evaluate if the HRP-crosslinked SF scaffolds support cartilage regeneration, and understand how the dynamic mechanical properties of constructs are affected after implantation, are potential areas of future works.

\section{Conclusions}

This study proposed novel HRP-crosslinked SF scaffolds developed in combination with the salt-leaching and freeze-drying technologies, for cartilage regeneration applications. These scaffolds presented suitable porosity and mechanical properties for cartilage scaffolding strategies, as well as, large swelling capacity and structural integrity after 30 days at physiological conditions. The scaffolds supported the attachment, viability and proliferation of human adipose-derived stem cells (hASCs) in vitro. The chondrogenic differentiation on the HRP-crosslinked SF scaffolds was represented by the peak of GAGs produced by the seeded hASCs after 21 days of chondrogenic culture. Additionally, the genotypic and phenotypic expression of chondrogenic-related markers, allied to the deposition of cartilage-specific ECM, also confirmed these observations. The HRP-crosslinked SF scaffolds maintained their original morphology and allowed tissue ingrowth when subcutaneously implanted in mice. Although complementary studies are necessary to biomechanically evaluate the anisotropic properties of the scaffolds, as well as, in vivo validate their efficacy and stability in articular cartilage defects, the results from scaffolds characterization, in vitro chondrogenic differentiation of hASCs at the HRP-crosslinked SF scaffolds and preliminary in vivo data, confirm that these can be promising structures for cartilage regeneration applications. Moreover, the multitude of structural, mechanical and biological parameters that can be achieved through these combined technologies can also encourage to extend their applicability for different TE strategies, including for osteochondral regeneration.

\section{Acknowledgments}

This study was funded by the Portuguese Foundation for Science and Technology (FCT) projects HierarchiTech and PEst (PEst- C/SAU/ LA0026/2013). The project FROnTHERA (NORTE-01-0145-FEDER000023), supported by Norte Portugal Regional Operational Programme (NORTE 2020), under the PORTUGAL 2020 Partnership Agreement, through the European Regional Development Fund (ERDF), is also greatly acknowledge. The authors are also grateful for the FCT distinctions attributed to J. M. Oliveira (IF/00423/2012 and IF/01285/2015), A. L. Oliveira (IF/00411/2013) and F. R. Maia (SFRH/BPD/117492/2016). V. P. Ribeiro (PD/BD/113806/2015) and J. Costa (PD/BD/113803/2015) were awarded PhD scholarships under the financial support from FCT/MCTES and FSE/POCH, PD/59/2013. R. Canadas was also awarded a FCT PhD scholarship (SFRH/BD/92565/2013). A. da Silva Morais acknowledge ERC2012-ADG 20120216-321266 (ComplexiTE) for his Post-doc scholarship. The authors also thank to Teresa Oliveira for the assistance with the histological preparation and to Le-Ping Yan for the valuable help with silk fibroin purification and hydrogels processing.

\section{Appendix A. Supplementary data}

Supplementary data associated with this article can be found, in the online version, at https://doi.org/10.1016/j.actbio.2018.03.047.

\section{References}

[1] E.A. Makris, A.H. Gomoll, K.N. Malizos, J.C. Hu, K.A. Athanasiou, Repair and tissue engineering techniques for articular cartilage, Nat. Rev. Rheumatol. 11 (1) (2015) 21-34

[2] W. Widuchowski, J. Widuchowski, T. Trzaska, Articular cartilage defects: study of 25,124 knee arthroscopies, The Knee 14 (3) (2007) 177-182.

[3] C.J. Moran, C. Pascual-Garrido, S. Chubinskaya, H.G. Potter, R.F. Warren, B.J Cole, S.A. Rodeo, Restoration of articular cartilage, JBJS 96 (4) (2014) 336-344.

[4] M. Brittberg, A. Lindahl, A. Nilsson, C. Ohlsson, O. Isaksson, L. Peterson, Treatment of deep cartilage defects in the knee with autologous chondrocyte transplantation, New Engl. J. Med. 331 (14) (1994) 889-895.

[5] J. Gille, J. Kunow, L. Boisch, P. Behrens, I. Bos, C. Hoffmann, W. Köller, M. Russlies, B. Kurz, Cell-laden and cell-free matrix-induced chondrogenesis versus microfracture for the treatment of articular cartilage defects: a histological and biomechanical study in sheep, Cartilage 1 (1) (2010) 29-42.

[6] J. Gille, P. Behrens, P. Volpi, L. De Girolamo, E. Reiss, W. Zoch, S. Anders, Outcome of Autologous Matrix Induced Chondrogenesis (AMIC) in cartilage knee surgery: data of the AMIC Registry, Arch. Orthopaedic Trauma Surg. 133 (1) (2013) 87-93.

[7] Y.P. Singh, N. Bhardwaj, B.B. Mandal, Potential of agarose/silk fibroin blended hydrogel for in vitro cartilage tissue engineering, ACS Appl. Mater. Interfaces 8 (33) (2016) 21236-21249.

[8] K.-S. Han, J.E. Song, N. Tripathy, H. Kim, B.M. Moon, C.H. Park, G. Khang, Effect of pore sizes of silk scaffolds for cartilage tissue engineering, Macromol. Res. 23 (12) (2015) 1091-1097.

[9] W. Zhao, X. Jin, Y. Cong, Y. Liu, J. Fu, Degradable natural polymer hydrogels for articular cartilage tissue engineering, J. Chem. Technol. Biotechnol. 88 (3) (2013) 327-339.

[10] B. Mollon, R. Kandel, J. Chahal, J. Theodoropoulos, The clinical status of cartilage tissue regeneration in humans, Osteoarthritis Cartilage 21 (12) (2013) 1824-1833.

[11] Y.J. Chuah, Y. Peck, J.E.J. Lau, H.T. Hee, D.-A. Wang, Hydrogel based cartilaginous tissue regeneration: recent insights and technologies, Biomater. Sci. 5 (4) (2017) 613-631. 
[12] P.H.G. Chao, S. Yodmuang, X. Wang, L. Sun, D.L. Kaplan, G. Vunjak-Novakovic, Silk hydrogel for cartilage tissue engineering, J. Biomed. Mater. Res. Part B: Appl. Biomater. 95 (1) (2010) 84-90.

[13] K. Makaya, S. Terada, K. Ohgo, T. Asakura, Comparative study of silk fibroin porous scaffolds derived from salt/water and sucrose/hexafluoroisopropanol in cartilage formation, J. Biosci. Bioeng. 108 (1) (2009) 68-75.

[14] Y. Wang, U.-J. Kim, D.J. Blasioli, H.-J. Kim, D.L. Kaplan, In vitro cartilage tissue engineering with 3D porous aqueous-derived silk scaffolds and mesenchymal stem cells, Biomaterials 26 (34) (2005) 7082-7094.

[15] U.-J. Kim, J. Park, C. Li, H.-J. Jin, R. Valluzzi, D.L. Kaplan, Structure and properties of silk hydrogels, Biomacromolecules 5 (3) (2004) 786-792.

[16] L.P. Yan, J.M. Oliveira, A.L. Oliveira, R.L. Reis, Core-shell silk hydrogels with spatially tuned conformations as drug-delivery system, J. Tissue Eng. Regen. Med. 11 (11) (2016) 3168-3177.

[17] M. Fini, A. Motta, P. Torricelli, G. Giavaresi, N.N. Aldini, M. Tschon, R. Giardino, C. Migliaresi, The healing of confined critical size cancellous defects in the presence of silk fibroin hydrogel, Biomaterials 26 (17) (2005) 3527-3536.

[18] X. Hu, K. Shmelev, L. Sun, E.-S. Gil, S.-H. Park, P. Cebe, D.L. Kaplan, Regulation of silk material structure by temperature-controlled water vapor annealing, Biomacromolecules 12 (5) (2011) 1686.

[19] K. Numata, T. Katashima, T. Sakai, State of water, molecular structure, and cytotoxicity of silk hydrogels, Biomacromolecules 12 (6) (2011) 2137-2144.

[20] T. Yucel, P. Cebe, D.L. Kaplan, Vortex-induced injectable silk fibroin hydrogels, Biophys. J. 97 (7) (2009) 2044-2050.

[21] X. Wang, J.A. Kluge, G.G. Leisk, D.L. Kaplan, Sonication-induced gelation of silk fibroin for cell encapsulation, Biomaterials 29 (8) (2008) 1054-1064.

[22] I. Karakutuk, F. Ak, O. Okay, Diepoxide-triggered conformational transition of silk fibroin: formation of hydrogels, Biomacromolecules 13 (4) (2012) 1122 1128.

[23] S.J. Sofia, A. Singh, D.L. Kaplan, Peroxidase-catalyzed crosslinking of functionalized polyaspartic acid polymers, J. Macromol. Sci., Part A 39 (10) (2002) 1151-1181.

[24] S. Sakai, I. Hashimoto, Y. Ogushi, K. Kawakami, Peroxidase-catalyzed cell encapsulation in subsieve-size capsules of alginate with phenol moieties in water-immiscible fluid dissolving H2O2, Biomacromolecules 8 (8) (2007) 2622-2626.

[25] L.-P. Yan, J. Silva-Correia, V.P. Ribeiro, V. Miranda-Gonçalves, C. Correia, A. da Silva Morais, R.A. Sousa, R.M. Reis, A.L. Oliveira, J.M. Oliveira, Tumor growth suppression induced by biomimetic silk fibroin hydrogels, Sci. Rep. 6 (2016) 31037.

[26] C.-H. Chen, J.M.-J. Liu, C.-K. Chua, S.-M. Chou, V.B.-H. Shyu, J.-P. Chen, Cartilage tissue engineering with silk fibroin scaffolds fabricated by indirect additive manufacturing technology, Materials 7 (3) (2014) 2104-2119.

[27] L.-P. Yan, J.M. Oliveira, A.L. Oliveira, S.G. Caridade, J.F. Mano, R.L. Reis, Macro microporous silk fibroin scaffolds with potential for articular cartilage and meniscus tissue engineering applications, Acta Biomater. 8 (1) (2012) 289 301.

[28] M. Marcacci, M. Berruto, D. Brocchetta, A. Delcogliano, D. Ghinelli, A. Gobbi, E. Kon, L. Pederzini, D. Rosa, G.L. Sacchetti, Articular Cartilage Engineering with Hyalograft (R) C: 3-Year Clinical Results, Clin. Orthopaedics Relat. Res. 435 (2005) 96-105.

[29] D.C. Crawford, C.M. Heveran, W. Dilworth Cannon, L.F. Foo, H.G. Potter, An autologous cartilage tissue implant NeoCart for treatment of Grade III Chondral injury to the distal femur, Am. J. Sports Med. 37 (7) (2009) 13341343.

[30] B.T. Estes, B.O. Diekman, J.M. Gimble, F. Guilak, Isolation of adipose-derived stem cells and their induction to a chondrogenic phenotype, Nat. Protoc. 5 (7) (2010) 1294-1311.

[31] A.I. Caplan, S.P. Bruder, Mesenchymal stem cells: building blocks for molecular medicine in the 21st century, Trends Mol. Med. 7 (6) (2001) 259-264.

[32] G.-I. Im, Y.-W. Shin, K.-B. Lee, Do adipose tissue-derived mesenchymal stem cells have the same osteogenic and chondrogenic potential as bone marrowderived cells?, Osteoarthritis Cartilage 13 (10) (2005) 845-853

[33] H.J. Kim, S.-H. Park, J. Durham, J.M. Gimble, D.L. Kaplan, J.L. Dragoo, In vitro chondrogenic differentiation of human adipose-derived stem cells with silk scaffolds, J. Tissue Eng. 3 (1) (2012). 2041731412466405.

[34] M. Naeimi, M. Rafienia, M. Fathi, M. Janmaleki, S. Bonakdar, M. EbrahimianHosseinabadi, Incorporation of chitosan nanoparticles into silk fibroin-based porous scaffolds: Chondrogenic differentiation of stem cells, Int. J. Polym. Mater. Polym. Biomater. 65 (4) (2016) 202-209.

[35] R.L. Horan, K. Antle, A.L. Collette, Y. Wang, J. Huang, J.E. Moreau, V. Volloch, D.L. Kaplan, G.H. Altman, In vitro degradation of silk fibroin, Biomaterials 26 (17) (2005) 3385-3393.

[36] M. Cerqueira, R.P. Pirraco, T. Santos, D. Rodrigues, A. Frias, A. Martins, R. Reis, A. Marques, Human adipose stem cells cell sheet constructs impact epidermal morphogenesis in full-thickness excisional wounds, Biomacromolecules 14 (11) (2013) 3997-4008.

[37] M. Dominici, K. Le Blanc, I. Mueller, I. Slaper-Cortenbach, F. Marini, D. Krause, R. Deans, A. Keating, D. Prockop, E. Horwitz, Minimal criteria for defining multipotent mesenchymal stromal cells, Int. Soc. Cell. Ther. Position Statement, Cytotherapy 8 (4) (2006) 315-317.
[38] K.J. Livak, T.D. Schmittgen, Analysis of relative gene expression data using realtime quantitative PCR and the $2-\Delta \Delta C T$ method, Methods 25 (4) (2001) 402-408.

[39] H.J. Jin, J. Park, V. Karageorgiou, U.J. Kim, R. Valluzzi, P. Cebe, D.L. Kaplan, Water-stable silk films with reduced $\beta$-sheet content, Adv. Funct. Mater. 15 (8) (2005) 1241-1247.

[40] P.J. Reardon, R. Konwarh, J.C. Knowles, B.B. Mandal, Mimicking hierarchical complexity of the osteochondral interface using electrospun silk-bioactive glass composites, ACS Appl. Mater. Interfaces 9 (9) (2017) 8000-8013.

[41] V.P. Ribeiro, J. Silva-Correia, A.I. Nascimento, A. da Silva Morais, A.P. Marques, A. S. Ribeiro, C.J. Silva, G. Bonifácio, R.A. Sousa, J.M. Oliveira, Silk-based anisotropical 3D biotextiles for bone regeneration, Biomaterials 123 (2017) 92-106.

[42] L.-P. Yan, J. Silva-Correia, M.B. Oliveira, C. Vilela, H. Pereira, R.A. Sousa, J.F. Mano, A.L. Oliveira, J.M. Oliveira, R.L. Reis, Bilayered silk/silk-nanoCaP scaffolds for osteochondral tissue engineering: in vitro and in vivo assessment of biological performance, Acta Biomater. 12 (2015) 227-241.

[43] H. Liu, H. Fan, Y. Wang, S.L. Toh, J.C. Goh, The interaction between a combined knitted silk scaffold and microporous silk sponge with human mesenchymal stem cells for ligament tissue engineering, Biomaterials 29 (6) (2008) 662-674.

[44] X. Zhang, C.B. Baughman, D.L. Kaplan, In vitro evaluation of electrospun silk fibroin scaffolds for vascular cell growth, Biomaterials 29 (14) (2008) 22172227.

[45] N. Bhardwaj, W.T. Sow, D. Devi, K.W. Ng, B.B. Mandal, N.-J. Cho, Silk fibroinkeratin based 3D scaffolds as a dermal substitute for skin tissue engineering, Integr. Biol. 7 (1) (2014) 53-63.

[46] R. Nazarov, H.-J. Jin, D.L. Kaplan, Porous 3-D scaffolds from regenerated silk fibroin, Biomacromolecules 5 (3) (2004) 718-726.

[47] G. Chen, T. Ushida, T. Tateishi, Scaffold design for tissue engineering, Macromol. Biosci. 2 (2) (2002) 67-77.

[48] U.-J. Kim, J. Park, H.J. Kim, M. Wada, D.L. Kaplan, Three-dimensional aqueousderived biomaterial scaffolds from silk fibroin, Biomaterials 26 (15) (2005) 2775-2785.

[49] H.J. Park, J.S. Lee, O.J. Lee, F.A. Sheikh, B.M. Moon, H.W. Ju, J.-H. Kim, D.-K. Kim, C.H. Park, Fabrication of microporous three-dimensional scaffolds from silk fibroin for tissue engineering, Macromol. Res. 22 (6) (2014) 592-599.

[50] D.J. Griffon, M.R. Sedighi, D.V. Schaeffer, J.A. Eurell, A.L. Johnson, Chitosan scaffolds: interconnective pore size and cartilage engineering, Acta Biomater. 2 (3) (2006) 313-320.

[51] L. Vikingsson, B. Claessens, J. Gómez-Tejedor, G.G. Ferrer, J.G. Ribelles, Relationship between micro-porosity, water permeability and mechanical behavior in scaffolds for cartilage engineering, J. Mech. Behav. Biomed. Mater. 48 (2015) 60-69.

[52] D. Yao, S. Dong, Q. Lu, X. Hu, D.L. Kaplan, B. Zhang, H. Zhu, Salt-leached silk scaffolds with tunable mechanical properties, Biomacromolecules 13 (11) (2012) 3723-3729.

[53] S. Chen, Y. Falcovitz, R. Schneiderman, A. Maroudas, R. Sah, Depth-dependent compressive properties of normal aged human femoral head articular cartilage: relationship to fixed charge density, Osteoarthritis Cartilage 9 (6) (2001) 561-569.

[54] L.R. Almeida, A.R. Martins, E.M. Fernandes, M.B. Oliveira, V.M. Correlo, I. Pashkuleva, A.P. Marques, A.S. Ribeiro, N.F. Durães, C.J. Silva, New biotextiles for tissue engineering: Development, characterization and in vitro cellular viability, Acta Biomater. 9 (9) (2013) 8167-8181.

[55] P. Gupta, M. Kumar, N. Bhardwaj, J.P. Kumar, C. Krishnamurthy, S.K. Nandi, B.B. Mandal, Mimicking form and function of native small diameter vascular conduits using mulberry and non-mulberry patterned silk films, ACS Appl. Mater. interfaces 8 (25) (2016) 15874-15888.

[56] L. Wu, J. Ding, Effects of porosity and pore size on in vitro degradation of threedimensional porous poly ( $D$, L-lactide-co-glycolide) scaffolds for tissue engineering, J. Biomed. Mater. Res. Part A 75 (4) (2005) 767-777.

[57] C. Csaki, P. Schneider, M. Shakibaei, Mesenchymal stem cells as a potential pool for cartilage tissue engineering, Ann. Anat. Anatomischer Anz. 190 (5) (2008) 395-412.

[58] N.-C. Cheng, B.T. Estes, H.A. Awad, F. Guilak, Chondrogenic differentiation of adipose-derived adult stem cells by a porous scaffold derived from native articular cartilage extracellular matrix, Tissue Eng. Part A 15 (2) (2008) 231241.

[59] M.K. Lotz, S. Otsuki, S.P. Grogan, R. Sah, R. Terkeltaub, D. D’lima, Cartilage cell clusters, Arthritis Rheumatol. 62 (8) (2010) 2206-2218.

[60] V. Dexheimer, S. Frank, W. Richter, Proliferation as a requirement for in vitro chondrogenesis of human mesenchymal stem cells, Stem Cells Dev. 21 (12) (2012) 2160-2169.

[61] F. Barry, R.E. Boynton, B. Liu, J.M. Murphy, Chondrogenic differentiation of mesenchymal stem cells from bone marrow: differentiation-dependent gene expression of matrix components, Exp. Cell Res. 268 (2) (2001) 189-200.

[62] D.K. Temple, A.A. Cederlund, B.M. Lawless, R.M. Aspden, D.M. Espino, Viscoelastic properties of human and bovine articular cartilage: a comparison of frequency-dependent trends, BMC musculoskeletal disorders 17 (1) (2016) 419.

[63] B. Kundu, R. Rajkhowa, S.C. Kundu, X. Wang, Silk fibroin biomaterials for tissue regenerations, Adv. Drug Delivery Rev. 65 (4) (2013) 457-470. 